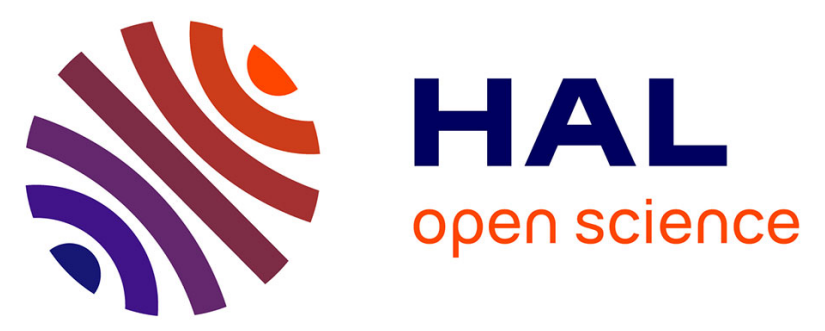

\title{
Allosteric inhibition of HER2 by Moesin-mimicking compounds targets HER2-positive cancers and brain metastases
}

Camille Faure, Rym Djerbi-Bouillié, Anaïs Domingot, Haniaa

Bouzinba-Segard, Saïd Taouji, Yanis Saidi, Sandra Bernard, Floriane Carallis, Romy Walther, Jean-Luc Lenormand, et al.

\section{To cite this version:}

Camille Faure, Rym Djerbi-Bouillié, Anaïs Domingot, Haniaa Bouzinba-Segard, Saïd Taouji, et al.. Allosteric inhibition of HER2 by Moesin-mimicking compounds targets HER2-positive cancers and brain metastases. Cancer Research, 2021, 10.1158/0008-5472.CAN-21-0162 . hal-03369875

\section{HAL Id: hal-03369875 \\ https://hal.archives-ouvertes.fr/hal-03369875}

Submitted on 7 Oct 2021

HAL is a multi-disciplinary open access archive for the deposit and dissemination of scientific research documents, whether they are published or not. The documents may come from teaching and research institutions in France or abroad, or from public or private research centers.
L'archive ouverte pluridisciplinaire HAL, est destinée au dépôt et à la diffusion de documents scientifiques de niveau recherche, publiés ou non, émanant des établissements d'enseignement et de recherche français ou étrangers, des laboratoires publics ou privés. 


\section{Allosteric inhibition of HER2 by Moesin-mimicking compounds targets HER2-positive cancers and brain metastases}

Camille Faure $^{1 *}$, Rym Djerbi-Bouillié ${ }^{1}$, Anaïs Domingot ${ }^{1}$, Haniaa Bouzinba-Segard ${ }^{1}$, Saïd Taouji ${ }^{2}$, Yanis Saidi ${ }^{1}$, Sandra Bernard ${ }^{1}$, Floriane Carallis ${ }^{1}$, Romy Walther ${ }^{3}$, Jean-Luc Lenormand ${ }^{3}$, Eric Chevet $^{2}$ and Sandrine Bourdoulous ${ }^{1 *}$

${ }^{1}$ Université de Paris, Institut Cochin, Inserm, CNRS, 75014 Paris, France

${ }^{2}$ Inserm, Université de Bordeaux, Institut Bergonié, 33000 Bordeaux, France

${ }^{3}$ TIMC-IMAG Laboratory, CNRS, Université Joseph Fourier, UFR de Médecine, 38700 La Tronche, France

* Corresponding authors:

Sandrine Bourdoulous and Camille Faure

Institut Cochin

22 rue Méchain

75014 Paris

France

Phone: +33140516427

sandrine.bourdoulous@inserm.fr

camille.faure@inserm.fr

Running title: Allosteric inhibition of HER2 as cancer treatment

Keywords: HER2-positive breast cancer/Moesin/ERM family/Brain metastases/Allosteric inhibition

The authors declare no potential conflicts of interest. 
CAN-21-0162R Faure et al

\section{ABSTRACT}

Therapies targeting the tyrosine kinase receptor HER2 have significantly improved survival of HER2+ cancer patients. However, both de novo and acquired resistance remain a challenge, particularly in the brain metastatic setting. Here we report that, unlike other HER tyrosine kinase receptors, HER2 possesses a binding motif in its cytosolic juxtamembrane region that allows interaction with members of the ezrin/radixin/moesin (ERM) family. Under physiological conditions, this interaction controls the localization of HER2 in ERM-enriched domains and stabilizes HER2 in a catalytically repressed state. In HER2+ breast cancers, low expression of moesin correlated with increased HER2 expression. Restoring expression of ERM proteins in HER2+ breast cancer cells was sufficient to revert HER2 activation and inhibit HER2-dependent proliferation. A high-throughput assay recapitulating the HER2/ERM interaction allowed for screening of about 1500 approved drugs. From this screen, Zuclopenthixol, an anti-psychotic drug that behaved as a moesin-mimicking compound, was found to directly bind the juxtamembrane region of HER2 and specifically inhibit HER2 activation in HER2+ cancers, as well as activation of oncogenic mutated and truncated forms of HER2. Zuclopenthixol efficiently inhibited HER2-positive breast tumor progression in vitro and in vivo and, more importantly, showed significant activity on HER2-positive brain tumor progression. Collectively, these data reveal a novel class of allosteric HER2 inhibitors, increasing the number of approaches to consider for intervention on HER2+ breast cancers and brain metastases.

Significance: This study demonstrates the functional role of moesin in maintaining HER2 in a catalytically repressed state and provides novel therapeutic approaches targeting HER2+ breast cancers and brain metastasis using moesin-mimicking compounds. 


\section{INTRODUCTION}

Human Epidermal Growth Factor Receptor 2-positive breast cancer (HER2+ breast cancer) is defined by amplification of the HER2/neu oncogene and/or overexpression of its associated HER2 transmembrane receptor protein (1). HER2+ breast tumours represent approximately $20-25 \%$ of breast cancers and are associated with a higher grade, more aggressive phenotype, and worse prognosis. With the advent of antibody-based (Trastuzumab, Pertuzumab) or small molecule tyrosine kinase inhibitors (Lapatinib) targeting HER2, the prognostic landscape for HER2+ breast cancer patients has considerably improved (2). However, both de novo and acquired resistance to trastuzumab and cardiotoxic side effects of these inhibitors remain a significant obstacle for extensive use of these treatments (3). About $30 \%$ of HER2+ breast tumours express a truncated form of HER2 (p95HER2), which confers resistance to trastuzumab and is associated with worse outcome in trastuzumab-treated patients $(4,5)$. More recently, somatic HER2 gene mutations were detected in a range of human cancer types that functionally activate HER2, drive and maintain cancers, and that can also confer resistance to HER2-directed drugs (6). Finally, these targeted therapies have limited ability to cross the bloodbrain barrier (BBB) and show low efficacy against brain lesions. Brain metastasis, occurring in up to $50 \%$ of the trastuzumab-treated patients, is an end in breast cancer progression (7), requiring novel therapies for successful treatment.

HER2 is a member of the HER family (EGFR/HER1, HER2, HER3, HER4) of transmembrane receptor tyrosine kinases (RTK) involved in various cellular processes including cell proliferation, motility, resistance to apoptosis, invasiveness, and angiogenesis. This RTK displays unique properties. Structurally, HER2 contains an extracellular domain (amino-acids 1-639) locked in an open conformation, a hydrophobic transmembrane domain (aa 640-672), a cytosolic juxtamembrane region (673-701), a tyrosine kinase domain (aa 720-977), and a C-terminal tail with regulatory tyrosine residues (978-1255) (8-10). A critical distinction defining its unique cell-signalling mode is its ability to promote its ligand-independent auto-dimerization and phosphorylation (11), as well as liganddependent heterodimerisation and phosphorylation with the other HER family members $(10,12)$. In HER2+ breast cancers, HER2/HER3 heterodimerisation promotes HER2 phosphorylation and phosphorylation of the kinase defective HER3, leading to stimulation of the downstream oncogenic PI3K/AKT pathway, while HER2 homodimerisation leads to downstream oncogenic RAS/MAPK and indirect PI3K/AKT pathway activation $(11,13,14)$.

ERM (Ezrin, Radixin, Moesin) proteins are involved in many cellular processes (15). These cytosolic proteins localise at cell surface-associated structures, such as microvilli and cell-adhesion sites (16). They link transmembrane proteins and the cortical cytoskeleton by interacting with a RxxTYxVxxA motif located in the juxtamembrane region of several adhesion molecules, such as the hyaluronic 
receptor CD44, through their $\mathrm{N}$-terminal FERM domain and by interacting through their $\mathrm{C}$-terminal domain with F-actin (17-19). They are critical players in cell migration, growth and adhesion, but also cell invasion and metastasis formation $(15,16)$.

Here, we found that HER2 possesses a binding motif in its intracellular juxtamembrane region allowing allosteric inhibition by members of the ERM family. In HER2+ breast cancers, low Moesin expression correlates with increased HER2 expression. Through a high content screen designed to identify Moesinmimicking compounds, we discovered Zuclopenthixol. This compound efficiently inhibited activation of oncogenic forms of HER2, specifically blocked HER2+ breast cancer progression both in vitro and in vivo. Finally, known to cross the blood-brain barrier, it attenuated the growth of HER2+ breast tumours following brain implantation. These findings reveal a novel class of allosteric HER2 inhibitors with activity on both breast- and brain-localised tumours, which could markedly improve the treatment of HER2+ breast cancer and metastases.

\section{MATERIALS AND METHODS}

\section{Reagents}

A list of the antibodies used in this work is provided in Supplementary Table 1.

Zuclopenthixol hydrochloride, Flupenthixol, and derived analogs were synthetized and purified by Roowin (Riom, France).

\section{Plasmids and mutagenesis}

The list of primers and siRNA used in this work is provided in Supplementary Table 2.

The vectors used in this work are detailed in the Supplementary Materials and Methods

\section{Cell culture and transfection}

HBMECs (human bone marrow endothelial cells) kindly provided by Dr. B. Weksler (Weill Medical College of Cornell University, NY) and Caco-2/TC7 cells kindly provided by Dr M. Rousset (Université Pierre et Marie Curie, Paris) were cultured as described previously (20), (21). The porcine kidney epithelial cell line LLC-PK1, the human breast cancer cell lines (SKBR3, BT474, MDAMB-231 and HCC1954) were obtained from the American Type Culture Collection (Manassas, VA) and were maintained in DMEM or RPMI-1640 (HCC1954) containing 10\% FBS. All cell lines were cultured under $5 \% \mathrm{CO} 2$ at $37^{\circ} \mathrm{C}$. HBMECs and LLC-PK1 were transfected using Amaxa Inc nucleofector system (Kit V and U015 program) or Biorad electroporation system, respectively as previously described $(22,23)$. SKBR3, BT474 and HCC1954 were transfected with Amaxa using Kit C and E009 program. Proliferation and soft agar colony formation assays are described in supplementary methods. Periodic tests for Mycoplasma and authentication were performed using commercially available kits. 


\section{Transcriptomic analyses}

HER2 and Moesin mRNA expression levels in patients with breast invasive carcinoma ( $n=526)$, stomach adenocarcinoma $(n=478)$, bladder urothelial carcinoma $(n=408)$, pancreatic adenocarcinoma $(n=178)$, colorectal adenocarcinoma ( $n=379)$, ovarian serous cystadenocarcinoma $(n=304)$, kidney renal clear cell carcinoma $(n=533)$ and kidney renal papillary cell carcinoma $(n=290)$ were from TCGA, Firehose Legacy. HER2 and Moesin mRNA levels in patients with cervical squamous cell carcinoma $(n=278)$ were from TCGA, PanCancer Atlas. mRNA data were z-scores relative to diploid samples (RNA Seq V2 RSEM) downloaded through cbioportal (http://www.cbioportal.org). Cancer cell lines mRNA expression data were obtained from E-MTAB-2706 study (https://www.ebi.ac.uk/arrayexpress/).

\section{Breast cancer tissue array}

Sections from breast cancer tissue array (FFPE) including IHC results of HER2 \ER\PR\Ki67 for 3 normal breast tissue and breast fibroadenoma, 2 breast cystosarcoma phyllodes, 7 breast intraductal carcinoma and 60 breast invasive ductal carcinoma, with duplicate cores per case were purchased from US Biomax, Inc (https://www.biomax.us/tissue-arrays/Breast/BR1503f). They were processed for immunohistochemistry using HER2, Ezrin or Moesin antibodies using a fully automated Leica (Hesse, Germany) BondIII stainer, according to the manufacturer's recommendations. Sections were then lightly counterstained with hematoxylin, dehydrated and cleared in graded alcohol and Ottix plus (MMFrance), and finally covered with glass slips. Samples were scanned using a Lamina multilabel slide scanner (PerkinElmer) and were classified based on visual scoring of Moesin-positive tumour cells as Moesin low (0 to $10 \%)$, intermediate (11-50\%) or high (>50\%) expressing samples.

\section{Tumour progression in orthotopic xenograft model}

Experiments were performed on the TrGET platform of centre de Recherche de Cancérologie de Marseille (Institut Paoli Calmettes, Marseille, France) in accordance with the guidelines of the Institut National de la Santé et de la Recherche Médicale. The experimental protocol was approved by the Animal Experimentation Ethics Committee of Marseille (APAFIS\#2079-2015092811101360 v3). Briefly, $5.10^{6}$ BT474 cells resuspended in $100 \mu \mathrm{l}$ (50\% matrigel and 50\% PBS) were implanted orthotopically in the mammary fat pad of NOD.Cg-Prkdc scid/J mice anesthetized with ketamine and xylazine and administered with metacam (SC, $1 \mathrm{mg} / \mathrm{kg}$ ). Estradiol pellets were implanted subcutaneously. Metacam was administered $24 \mathrm{~h}$ and $48 \mathrm{~h}$ post implantation. Nineteen days after implantation, mice were randomized into 3 groups to be administered intraperitoneally during three weeks with vehicle ( $10 \%$ DMSO in PBS, 5 days a week, $\mathrm{n}=9$ ), $4 \mathrm{mg} / \mathrm{kg}$ Zuclopenthixol ( 5 days a week, $\mathrm{n}=8$ ) or $5 \mathrm{~m} / \mathrm{kg}$ Zuclopenthixol ( 3 days a week, $n=9$ ). Mice weight and tumour volume were assessed 2 to 3 times a week using a calliper. At the end of the experiment, the collected tumours were photographed, weighed, cut into halves to be either frozen in liquid nitrogen or embedded in OCT and frozen at $-80^{\circ} \mathrm{C}$. 
A tissue Lyzer ${ }^{\mathrm{TM}}$ was used to solubilize tumour proteins in a buffer containing $50 \mathrm{mM}$ Tris $\mathrm{pH}=8.8,25$ $\mathrm{mM} \mathrm{NaCl}, 1 \mathrm{mM}$ EDTA, 1\% Triton, 10\% glycerol and $1 \mathrm{mM}$ phosphatase (AEBSF and $1 \mathrm{mM}$ orthovanadate) and $10 \mu \mathrm{g} / \mathrm{mL}$ protease inhibitors (aprotinin, leupeptin, pepstatin). $5 \mu \mathrm{m}$ thick sections were immobilised on superfrost ${ }^{\mathrm{TM}}$ plus microscope slides and proceeded for immunofluorescence or immunochemistry analysis. Samples were scanned using a Lamina multilabel slide scanner (PerkinElmer) and were further analysed using confocal microscopy (spinning disk Leica, 40x). For quantitative analysis, fluorescence intensity of KI67 and DAPI labelling were measured using ImageJ software $(\mathrm{NIH})$ and the proliferation index is displayed as a ratio between KI67-stained area over DAPIstained area.

\section{Tumour progression in intracranial xenograft model}

Experiments were performed by Oncodesign contract research organization (Dijon, France). The experimental protocol was approved by the Animal Experimentation Ethics Committee OnCoMet ( $\mathrm{n}^{\circ}$ study: 180078ET100). Briefly, $1.10^{5}$ BT474 cells resuspended in $2 \mu \mathrm{L}$ RPMI 1640 medium were stereotactically implanted into the caudate nucleus of the right cerebral hemisphere of 25 female BALB/c Nude mice, 4-5 weeks old, under gas anaesthesia with isoflurane. Drinking water was supplemented with estradiol $(2.5 \mu \mathrm{g} / \mathrm{mL})$. In the first set of experiments, magnetic resonance imaging (MRI) was performed at day 10, mice were then randomized and intraperitoneally received Zuclopenthixol ( $\mathrm{n}=10,7 \mathrm{mg} / \mathrm{kg}$ on Monday and Tuesday and $10 \mathrm{mg} / \mathrm{kg}$ on Wednesday, Thursday and Friday) or vehicle ( $n=10,20 \% \mathrm{w} / \mathrm{v}$ hydroxypropyl- $\beta$-cyclodextrin per day for 5 consecutive days a week) and a second MRI was performed at day 20. In the second set of experiments, implanted mice were randomized at day 3 and intraperitoneally received Zuclopenthixol, vehicle at doses identical to the first set or received lapatinib (100 mg/kg, PO, N=10). MRI were performed at days 15 and 26 postimplantation. During MRI, mice were continuously anesthetized using isoflurane. Gadopentetate dimeglumine (Gd-DTPA, Magnevist, Bayer Healthcare Pharmaceuticals, Germany) was injected intravenously at $0.4 \mathrm{mmol} / \mathrm{kg}$. MRI were acquired on a 4. 7T horizontal magnet (PharmaScan, Bruker Biospin $\mathrm{GmbH}$, Germany) equipped with an actively shielded gradient system with the following parameters: time of repetition $=700 \mathrm{~ms}$, echo time $=12 \mathrm{~ms}$, field of view $=40 \mathrm{~mm} \times 40 \mathrm{~mm}$, acquisition matrix $=384 \times 384$, slice thickness $=0.8 \mathrm{~mm}$, slice gap $=0.8 \mathrm{~mm}$, slice number $=10$ and number of averages $=4$. The positions of the slices were determined using sagittal, coronal and axial imaging. MRI images were acquired under Para Vision (PV5.1, Bruker Biospin) and analysed under ImageJ. Regions of interest were drawn manually on anatomical images. Tumour volume was computed from these regions by multiplying the number of voxels by the voxel volume (in $\mathrm{mm}^{3}$ ). 


\section{Statistical analysis}

Statistical analyses were performed by GraphPad Prism 5 software. Unpaired/paired Student $t$ test was used to analyze the statistical difference of two groups. One- or two-way ANOVA test followed by multiple comparison test, as indicated, was used to analyze the statistical difference between multiple groups. The correlation between HER2 and ERM expression was analyzed by Spearman $r$ correlation coefficient. Data are presented as the mean \pm SD or sem as indicated. $P$ value $<0.05$ was considered statistically significant.

Additional Materials and Methods are provided in the Supplementary Information.

\section{RESULTS}

\section{ERM proteins Ezrin and Moesin interact with HER2 and address HER2 to ERM-enriched regions}

A direct interaction between the FERM domain of the ERM proteins and a R/KxxxYxL/V/IxxA motif located in the cytosolic juxtamembrane regions of InterCellular Adhesion Molecules (ICAM-1,-2, -3) CD43 and CD44 was previously reported $(17,19)$. We identified in the cytosolic juxtamembrane region of HER2 a similar ERM binding motif between Lys-676 and Leu-691, that was absent in other EGFR family members (Fig. 1A). To address whether this motif also allows interaction of HER2 with the ERM proteins, we generated a glycoprotein D-tagged HER2 form harbouring mutations in the critical amino acid residues involved in this ERM binding motif (gD-HER2-EBM*) (Fig. 1A). When ectopically expressed in human endothelial cells (HBMEC), the wild type gD-tagged form of the receptor (gD-HER2-WT) coimmunoprecipitated with Ezrin and Moesin (Fig. 1B). Although gD-HER2-EBM* construct was immunoprecipitated to a lower extent, interaction was drastically reduced with this construct (Fig. 1B). In addition, both endogenous HER2 (Sup Fig. 1A) and gD-HER2-WT (Fig. 1C) were efficiently pulled down by the FERM domain of Ezrin fused to GST (GST-FERME) but not with GST alone, while gD-HER2EBM* was poorly pulled down with GST-FERM $($ Fig. 1C), demonstrating weak binding of this mutated form to the FERM domain of Ezrin.

To investigate whether this interaction was direct, we first used a protein-protein interaction assay based on oxygen singlet transfer (AlphaScreen ${ }^{\circledR}$ ). We detected an association between the biotinylated peptide encoding the cytosolic juxtamembrane region of HER2 (biot-JM $\mathrm{HER}_{2}$ ) and GST-FERME, whereas a low signal was generated using GST alone or in the absence of any peptide (Fig. 1D). This interaction was dose-dependent (Sup Fig. 1B and 1C). Addition of non-biotinylated peptide coding for the

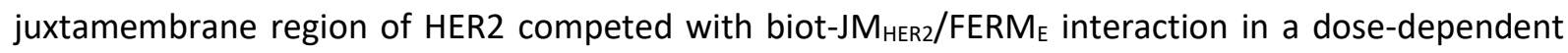
manner [maximum competition effect of $80 \%$, apparent $\mathrm{Kd}(\mathrm{Kda})=13 \mathrm{nM}$ ], whereas competition was poorly efficient upon addition of non-biotinylated HER2 peptide harbouring mutations of the key 
residues in the EBM motif ( $45 \%$ inhibition, $K d a=67 \mathrm{nM}$ ) (Sup Fig. 1D). The biot-JM HER2 $/ \mathrm{FERM}_{\mathrm{E}}$ interaction was in the same range of affinity as the interaction measured between GST-FERME and a biotinylated peptide coding for the cytosolic juxtamembrane region of CD44 (biot-JM $\mathrm{CD}_{44}$ ) containing a known ERM binding motif (Kda = $8 \mathrm{nM}$ ) (Sup Fig. 1E-G). Second, using surface plasmon resonance (SPR), we measured a high affinity interaction between GST-FERM $\mathrm{E}_{\mathrm{E}}$ and immobilized biot-JM $\mathrm{HER2}_{\text {2 }}$ peptide (Kd $=4.49 \pm 1.52 \mathrm{nM}$ ) (Fig. 1E), which was similar to the one observed with immobilized biot-JM $\mathrm{MD} 44_{4}$ peptide $(\mathrm{Kd}=5.79 \pm 0.12 \mathrm{nM})$ (Sup Fig. $1 \mathrm{H}$ ). No interaction was observed with GST alone or on immobilized peptide coding for the cytosolic region of $\mathrm{CD} 147\left(\right.$ (cyto $\left._{\mathrm{CD} 147}\right)$, a receptor which does not interact with ERM, as negative control (Fig. 1E). These results demonstrate that HER2 contains an ERM binding motif in its cytosolic juxtamembrane region allowing direct interaction with the FERM domain of ERM proteins.

In polarized epithelial cells, such as Caco2/TC7 intestinal epithelial cells, HER2 mostly localised at the basolateral membrane (Fig. 1F, top panels), a localisation resulting from the interaction of its Cterminal motif with PDZ domain-containing proteins $(24,25)$. A bipartite sorting signal (aa 692-701) next to the ERM binding motif is also necessary to ensure its proper targeting to the basolateral membrane (26). Less documented is the apical membrane localisation of HER2. We observed here that HER2 localises at the apical surface of Caco2/TC7 cells within ERM-enriched microvilli (Fig. 1F, top panels). When expressed in LLC-PK1 epithelial cells, which are deficient in the $\mu 1 \mathrm{~B}$ subunit of the AP1B adaptor complex, leading to significant amounts of HER2 that are missorted to the apical membrane (26), both gD-HER2 WT and gD-HER2-EBM* were found at the lateral junctions, however only the WT form of HER2 co-localised with Ezrin within apical microvilli (Fig. 1F, bottom panels), indicating that HER2 interaction with ERM proteins controls the subcellular localisation of HER2 within ERM-enriched structures.

\section{ERM proteins control the ligand-independent activation of HER2}

We next observed that, when overexpressed in HBMEC, gD-HER2-EBM* exhibited higher levels of tyrosine phosphorylation compared to gD-HER2-WT (Sup Fig. 2A), suggesting that loss of HER2/ERM interaction might potentiate receptor activation. To test this hypothesis, we overexpressed gD-HER2WT in HBMEC with GFP-tagged Ezrin or Moesin. The phosphorylation level of HER2 was reduced by more than $75 \%$ when co-expressed with Ezrin or Moesin (Fig. 1G). Expression of Ezrin $\left(\mathrm{FERM}_{\mathrm{E}}\right.$ ) or Moesin $\left(F E R M_{M}\right)$ FERM domains alone was sufficient to reduce the phosphorylation level of overexpressed HER2 (Fig. 1H and Sup Fig. 2B). To confirm this result, we overexpressed HER2 with VSVg-tagged Ezrin or with two previously described VSVg-tagged mutants of Ezrin: the T567D mutant mimicking the phosphorylation of a conserved threonine in the C-terminal actin binding site, which stabilises Ezrin in an active open conformation, and the non-phosphorylable mutant T567A $(22,27)$, or 
GFP as a negative control. The phosphorylation level of HER2 was reduced by 20 and $30 \%$ when coexpressed with Ezrin or with the T567A Ezrin mutant, and reduced by 55\% when co-expressed with the T567D Ezrin mutant (Sup Fig. 2C). HER2 inhibition promoted by these forms of Ezrin was accompanied by a proportional reduction in the downstream activation of ERK and AKT protein kinases (Fig. 1G and Sup Fig. 2C). Accordingly, expression of gD-HER2-WT induced a 2 to 3 -fold increase in the rate of endothelial cell proliferation, which was reduced by 70 to $85 \%$ when co-expressed with FERM $M_{E}$, whereas FERM $M_{E}$ did not affect the basal cell proliferation (Fig. 1I). As expected, expression of FERME had no effect on HER2-EBM* activation (Fig. 1H), nor on HER2-EBM*-induced cell proliferation (Fig. 1I). In contrast, expression of the FERM domains of FAK or PYK2 (FERM $M_{F}$ and FERMP) had no effect despite their related structure but lesser sequence similarity (Sup Fig. 2B), indicating that this inhibitory effect was specific to the ERM proteins.

Conversely, depletion of Ezrin, Moesin, or both in HBMEC induced respectively a 2, 3 and 4-fold increase in activation of endogenous HER2, together with a 3-fold increase in AKT activation, whereas depletion of the actin binding protein cortactin, as a control, did not affect HER2 activation (Fig. 1J). Accordingly, depletion of Ezrin and Moesin further increased HER2 activation and the proliferation rate of endothelial cells expressing gD-HER2-WT, while it had no further effect on HER2-EBM* activation, nor on HER2-EBM*-induced cell proliferation (Sup Fig. 2D and 2E). These experiments showed that Ezrin and Moesin exert a constitutive inhibitory effect that prevents the ligand-independent activation of HER2. However, although HER2 is the preferential heterodimerisation partner of the other HER family members $(10,12)$, expression of the Ezrin-VSV-g or of FERM $E$ did not alter the ligand-dependent activation of HER2 in heterodimers with EGFR/HER1 or with HER3 upon EGF or HRG stimulation, nor downstream activation of AKT and ERK MAPK (Sup Fig. 2F and 2G). These results demonstrate that ERM proteins are allosteric inhibitors of HER2 by interacting with the ERM binding motif present in its juxtamembrane region. This interaction specifically prevents the ligand-independent activation of HER2.

\section{The unbalance in ERM/HER2 expression contributes to HER2 activation in HER2+ breast cancers}

Interestingly, we observed a significant inverse correlation between HER2 and Moesin transcripts ( $r=-$ $0.2866, \mathrm{P}=0.0161$ ) in 70 commonly used human breast cancer cell lines, whereas a positive or no correlation between HER2 and Ezrin or Radixin mRNA expression was observed (Ezrin: $r=0.3386, P=$ 0.0041; Radixin: $r=-0.1509, P=0.2123$ ) (Fig. $2 A$ ). At the protein level, we also detected an inverse correlation between HER2 and Moesin expression in breast cancer cell lines with different HER2 status, while they expressed similar levels of Ezrin and Radixin (Fig 2B and Sup Fig. 3A-B). A significant inverse correlation between Moesin and HER2 mRNA expression levels (Spearman correlation coefficient=0.3010; $\mathrm{P}<0.0001$ ) was also observed in 526 patients-derived breast invasive carcinoma (Fig. 2 C). 
Among ERM protein members, a negative correlation with HER2 expression was also seen with Radixin, whereas a positive correlation was found with Ezrin (Ezrin: $r=0.1538, P=0.0004$; Radixin: $r=-0.1783$, $\mathrm{P}<0.0001$ ) (Fig. 2C). Likewise, immunohistochemistry analysis performed on tissue microarray containing 67 ductal carcinomas with different HER2 status revealed a significant inverse correlation between Moesin and HER2 protein expression levels, while Ezrin remains expressed in HER2+ breast cancers (Fig. 2D and Sup Fig. 4A-C). Finally, similar observation was found in most cancer types known to have abnormalities in ERBB2 gene (cervix, stomach, bladder, pancreas, colon, ovary and kidney) (Sup Fig. 5). Altogether, these results indicated that low Moesin expression was significantly associated with HER2+ cancers and, based on our previous results, we hypothesized that this might contribute to aberrant activation of HER2.

To test this hypothesis, we overexpressed Moesin, Ezrin or their respective FERM domains in SKBR3 or BT474, two breast cancer cell lines overexpressing HER2 and devoid of Moesin expression (Fig. 2B). Their overexpression reduced the aberrant activation of HER2 by $60 \%$ in BT474 (Fig. 2E) and by $40 \%$ and $100 \%$ respectively in SKBR3 cells (Sup Fig. 6A). This effect was accompanied by decreased AKT activation (Sup Fig. 6A) and inhibition of cell proliferation (Fig. 2F and Sup Fig. 6B-C). Interestingly, in HCC1954 breast cancer cell line expressing Moesin, although HER2 expression level was similar to SKBR3 and BT474 cells, HER2 activation was lower in these cells (Fig. 2B). The overexpression of Ezrin, FERME $_{\mathrm{E}}$ or Moesin in HCC1954 totally inhibited cell proliferation (Sup Fig. 6D-E). To strengthen this result, we fused FERM , or GFP as a control, to a cell-penetrating peptide derived from the EpsteinBarr virus ZEBRA transcription factor (28) (Sup Fig. 6F). As expected, incubation of SKBR3 cells with 0.3 to $0.9 \mu \mathrm{M}$ FERME-Zebra induced a dose-dependent inhibition of HER2 activation up to $85 \%$, whereas Zebra-GFP did not modify HER2 activation (Sup Fig. 6G). Altogether, these results demonstrate that ERM proteins are key allosteric regulators of HER2 activation. The unbalance in ERM/HER2 protein levels contributes to HER2 activation in HER2+ breast cancers.

\section{Identification of ERM-mimicking compounds to inhibit HER2 activation}

As overexpression of ERM proteins efficiently reverted HER2 activation in HER2+ breast cancers, we designed a strategy aimed at identifying ERM mimicking compounds that would actively block HER2 (Fig. 3A). Compounds were first selected on their ability to interfere with the HER2/FERME interaction measured using the above-described AlphaScreen ${ }^{\circledR}$ assay (Fig. 1D). After removal of the false positives (True hit screen), to eliminate compounds interfering with the HER2/FERM $\mathrm{E}_{\mathrm{E}}$ interaction through binding to GST-FERM $\mathrm{E}_{\mathrm{E}}$, we performed a counter-screening AlphaScreen ${ }^{\circledast}$ assay to identify hits showing competition with the CD44/FERME interaction. The remaining hits were then characterized for their ability to directly bind to the ERM binding motif of HER2 using SPR, to inhibit HER2 activation and HER2dependent cell proliferation and for their absence of toxicity on cells that do not overexpress HER2. 
Finally, the best hits were tested in vivo in an orthotopic xenograft model to evaluate their ability to inhibit HER2-dependent tumour growth. 1463 molecules were screened from 2 Prestwick chemical libraries consisting of small molecules composed mostly of approved drugs selected for their high chemical and pharmacological diversity, as well as for their known bioavailability and safety in humans, and a collection of natural products, mostly derived from plants, rich in diverse chemotypes. Results of the first screen at $24 \mathrm{~h}$ are shown in Fig. $3 \mathrm{~B}$. Among the 77 compounds showing inhibition of

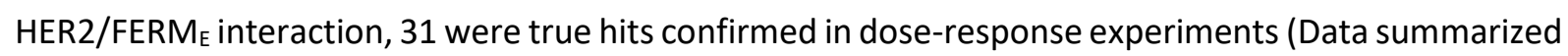
in Table 1). Interestingly, some compounds, such as tolfenamic acid and 4,4'-(2,3Dimethyltetramethylene) dipyrocatechol, were already known to block the growth of HER2+ cancer cells $(29,30)$. Eleven hits were found to selectively interfere with $H E R 2 / F E R M_{E}$ interaction and not with

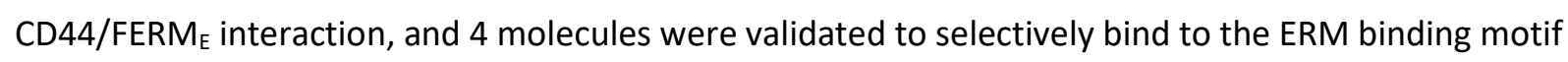
of HER2 using SPR. Among them, phenylbutazone and ebselen induced a poor inhibition of HER2 activation, isoliquiritigenin inhibited HER2 activation but, as it possessed genotoxic structural alerts, it was unsuitable for drug discovery. Finally, Zuclopenthixol hydrochloride was found to achieve potent HER2 inhibition both in vitro and in vivo (results detailed below).

\section{Zuclopenthixol is an ERM-mimicking compound conferring efficient allosteric inhibition of HER2}

In vitro, Zuclopenthixol induced a dose-dependent inhibition of the HER2/FERME interaction reaching $87 \%\left(\mathrm{IC}_{50}=9.8 \mu \mathrm{M}\right)$ at $24 \mathrm{~h}$ with no or low disruption of CD44/FERME interaction (Table 1$)$. Zuclopenthixol selectively bound to the ERM binding motif of HER2 as assessed using SPR (Sup Fig. 7A) and induced a dose-dependent inhibition of gD-HER2-WT overexpressed in HBMEC, accompanied by a proportional reduction in the activation of AKT and ERK protein kinases, whereas, as expected, it had no effect on gD-HER2-EBM* activation, nor on gD-HER2-EBM*-induced activation of AKT and ERK protein kinases (Sup Fig 7B). Zuclopenthixol also promoted a dose-dependent inhibition of HER2 activation in SKBR3 cells (Sup Fig 7C). Treatment with 3-5 $\mu \mathrm{M}$ Zuclopenthixol reduced by $45 \%$ and $55 \%$ the cell proliferation of SKBR3 or BT474, respectively, whereas no effect was observed on the proliferation of breast cancer cells that do no overexpress HER2 (MDAMB-231) (Fig. 3C and Sup Fig. 7D), demonstrating that this compound selectively inhibits HER2-dependent cell proliferation. Finally, as expected HCC1954 were also sensitive to the inhibitory effect of Zuclopenthixol, although they express Moesin (Sup Fig. 7E).

We then tested the effect of Zuclopenthixol on the anchorage-independent proliferation of breast cancer cell lines, as it correlates closely with tumorigenicity in animal model (31). Treatment with $5 \mu \mathrm{M}$ Zuclopenthixol induced a 50\% decrease in the size of SKBR3 micro-colonies (Fig. 3D) and completely blocked the development of micro-colonies formed by BT474 cells (Fig. 3E), whereas it had no effect on the development of MDAMB-231 micro-colonies (Fig. 3F). As a positive control, AG1478, a non- 
specific HER2 kinase inhibitor, induced a 75\% decrease in the size of SKBR3 micro-colonies, whereas it had no effect on the formation of MDAMB-231 micro-colonies (Sup Fig. 7F). These results demonstrate that Zuclopenthixol selectively inhibits the anchorage-independent proliferation of breast cancer cell lines overexpressing HER2.

Zuclopenthixol is a typical antipsychotic from the thioxanthene family (commercialized as Clopixol, Cisordinol, or Acuphase) that acts by blockade of dopamine receptors. Similar results were observed with the related thioxanthene molecule Flupenthixol (Sup Table 3). However, poor or no effects were observed with other closely related dopamine antagonists, such as antipsychotics of the thioxanthene family (Chlorprothixene) or phenothiazine family (Thioproperazine) (Sup Table 3). Finally, close analogs of Zuclopenthixol were synthetized. Analog 1 inhibited the HER2/FERME interaction in vitro, HER2 activation and the anchorage-independent growth of SKBR3, although less efficiently than Zuclopenthixol and Flupenthixol, whereas no significant effects were observed with Analogs 2 and 3 (Sup Table 3). These data indicate that Zuclopenthixol and Flupenthixol were the most effective compounds in vitro, and that they promote blockade of HER2 by a mechanism independent of their neuroleptic activity.

As expected, Zuclopenthixol also efficiently inhibited HER2 activation, as well as both the anchoragedependent and -independent proliferation of gastric ( $\mathrm{NCl}-87$ ) and ovary (SKOV3) cell lines overexpressing HER2, demonstrating its potent action on HER2+ cancers (Sup Fig. 8A-C). Finally, Zuclopenthixol induced a dose-dependent inhibition of the truncated form of HER2 (p95HER2), which is resistant to antibody-based therapy, accompanied by a reduction in activation of AKT and ERK protein kinases (Sup Fig. 8D), and inhibited cell proliferation induced by the expression of mutated forms of HER2, V777L and V842I, two activating mutations in the kinase domain found in cancer patients and conferring resistance to tyrosine kinase inhibitors (Sup Fig. 8E). These results demonstrate that, by targeting the ERM binding motif in the juxtamembrane region of HER2, Zuclopenthixol may confer advantages over existing HER2-targeted therapies.

\section{Zuclopenthixol inhibits the HER2-dependent tumour growth in vivo}

To explore the potential of Zuclopenthixol to reduce the growth of HER2-overexpressing tumours in vivo, we used BT474 cells orthotopically implanted in the mammary fat pad of immunodeficient NOG mice (Fig. 4A). Treatment with Zuclopenthixol (4mg/kg, 5 days a week), or vehicle as a control, was initiated 19 days after cell engraftment. While control tumours followed an exponential growth curve, Zuclopenthixol treatment rapidly slowed down the tumour growth, to reach a meaningful tumour growth inhibition index of $40 \% 10$ days post-treatment (Day 29), with a sustained effect until the Day 40 (Fig. 4B and 4C). Accordingly, tumours from Zuclopenthixol-treated mice were macroscopically smaller (Fig. 4D) and exhibited a 40\% reduction in tumour weight compared to the control $(P=0.0196)$ 
(Fig. 4E). Zuclopenthixol treatment was overall well tolerated as indicated with the monitoring of mice body weight (Fig. 4F). Similar results were obtained with another scheme of Zuclopenthixol administration ( $5 \mathrm{mg} / \mathrm{kg}, 3$ days a week), with a tumour growth inhibition index of 30\% at Day 29 (Fig. $4 B$ and $4 C$ ) and a $35 \%$ reduction in tumour weight compared to the control ( $P=0.0354$ ) (Fig. 4C and 4D). Biochemical analyses revealed a $45 \%$ decrease in HER2 activation in tumour lysates from Zuclopenthixol-treated animals compared to vehicle-treated animals, accompanied by a reduction in the activation of ERK protein kinases (Fig. $4 \mathrm{G}$ and $4 \mathrm{H}$ ). In agreement with a tumour growth inhibitory effect, Zuclopenthixol decreased by $85 \%$ the fraction of Ki-67-positive tumour cells (Fig. $4 \mathrm{I}$ and $4 \mathrm{~J}$ ). Altogether, these results provide strong evidence of the potent HER2 inhibitory effect of Zuclopenthixol on human breast cancer cells overexpressing HER2 in vivo.

\section{Zuclopenthixol reduces the growth of HER2-dependent brain tumours}

A major issue in the management of HER2+ breast cancers remains the disease progression in the central nervous system, since 30 to $50 \%$ of patients will develop brain metastases. This high incidence mainly results from the limited ability of actual therapies to cross the BBB (32) and the blood-tumour barrier remains largely intact (33). Because Zuclopenthixol is an antipsychotic with a known ability to cross the BBB, we addressed its capacity to reduce the growth of BT474 cells implanted in the caudate nucleus of the right hemisphere of immunodeficient Nude mice. Day 10 after intracranial implantation, mice were treated with Zuclopenthixol ( $M$, Tu: $7 \mathrm{mg} / \mathrm{kg} ; \mathrm{W}$, Th, F: $10 \mathrm{mg} / \mathrm{kg}$; IP; $\mathrm{n}=10$ ) or with vehicle $(I P, n=10)$ (Fig. 5A). At Day 20, brain tumours from the vehicle-treated group were of $75 \mathrm{~mm}^{3}$ average size, representing a 24.3-fold increase in their growth compared to Day 10. Zuclopenthixol administration was associated with smaller tumours $\left(50 \mathrm{~mm}^{3}\right)$ exhibiting significantly slower progression with a 14-fold increase in their growth compared to day 10 , therefore demonstrating a real benefit of Zuclopenthixol administration on HER2+ brain tumour progression (Fig. 5B-D). We next compared the efficiency of Zuclopenthixol (M, Tu: $7 \mathrm{mg} / \mathrm{kg}$; W, Th, F: $10 \mathrm{mg} / \mathrm{kg}$; IP; n=10) over Lapatinib (100 mg/kg 5d/w; PO; n=10) (Sup Fig. 9A). Treatments were initiated day 3 post-implantation. At Day 16 and Day 26, while Lapatinib had no effect as compared to control mice that received vehicle, Zuclopenthixol had reduced tumour size by 35 to $50 \%$ as compared to Lapatinib-treated tumours (Sup Fig. 9B-E). Combined, these data indicate that Zuclopenthixol would be promising as a therapeutic agent for HER2+ breast cancer with brain metastasis. 


\section{DISCUSSION}

Much research has been conducted in recent years to improve the treatments for HER2+ breast cancer, especially in light of incomplete action, adverse effects and de novo or acquired resistance to HER2 targeted therapies. This implies the development of pharmaceuticals that act on mutated and truncated forms of the receptor and on brain metastatic disease, the incidence of which is increasing due to the improved management of systemic disease and increased survival rates. This study revealed an inhibitory mechanism of HER2 by the ERM proteins and the translation of this discovery into the identification of a novel class of anti-HER2 inhibitors targeting the cytosolic juxtamembrane region of HER2. Among the molecules identified, the antipsychotic drug Zuclopenthixol demonstrated a potent action on the progression of human breast cancers overexpressing HER2, on cancer cells expressing mutated or truncated forms of HER2 and, due its ability to penetrate through the BBB, on HER2+ breast cancer-derived brain tumours. Overall, this inhibitor, via its mode of action, presents several advantages over existing anti-HER2 therapies and could thereby represent an important additional option in future HER2-targeted cancer therapy, in particular for brain tumour progression.

Among the large family of RTKs, HER2 displays unusual structural features conferring unique properties. Here, we have highlighted a novel structural particularity of this receptor, which, unlike other RTK family members, possesses a functional R/KxxxYxL/V/IxxA motif allowing interaction with ERM proteins (17-19). We further demonstrated that this interaction tightly controls HER2 localisation and activation. ERM proteins link membrane proteins to the cortical actin cytoskeleton, and therefore are known as key regulators of receptor localisation, trafficking and/or recycling at the cell plasma membrane thus controlling their surface availability and signalling (34). To date, direct interaction of ERM proteins with transmembrane proteins has only been described for adhesion molecules (18). The associations described between ERM proteins and several signalling receptors, including EGFR, occur indirectly through interaction with the adapter NHERF-1 (or ERM-binding phosphoprotein 50), that binds to the C-terminal sequence of these receptors $(34,35)$. The subcellular localisation of HER2 is tightly regulated by different mechanisms, such as interaction with specific protein partners. For instance, the co-localization of HER2 with PMCA2 (plasma membrane ATPase2) in actin-rich membrane domains seems required for its stabilisation at the plasma membrane (36). By interacting with NHERF1, Ezrin may also help membrane retention of the HER2-PMCA2-NHERF-1 complex $(37,38)$. The interaction of the PDZ domain ERBIN to the C-terminal part of HER2 has a critical role in restricting this receptor to the basolateral membrane of epithelial cells (24). Here, we demonstrated that, by interacting directly with HER2, ERM proteins redirect HER2 receptor within ERM-rich regions. Interestingly, a basolateral targeting signal able to redirect the apically localised receptor to the basolateral membrane domain of polarized epithelial cells has also been described in the amino acid 
sequence following ERM binding motif (26). Hence, our work, together with other studies, demonstrates that the cytosolic juxtamembrane region of HER2 plays a key role in the regulation of its subcellular distribution.

Previous investigations have also shown that the juxtamembrane region plays a crucial role in the activation of HER receptors upon ligand binding, by stabilizing the formation of asymmetric kinase domain dimers allowing the transphosphorylation process (39-41). Despite the unique structural conformation of the extracellular domain of HER2 locked in an open and active conformation (10), HER2 remains in inactive homodimers unless overexpressed (42) suggesting an endogenous mechanism able to restrain its ligand-independent activation $(40,41)$. Here, we provide evidence that interaction of ERM proteins with the juxtamembrane region of HER2 prevents this ligand-independent activation. Accordingly, loss of ERM protein expression or mutations in the ERM binding motif of HER2 both enhanced HER2 activity. Furthermore, recurrent somatic mutations in the HER2 juxtamembrane domain were found in different patient tumours (https://cancer.sanger.ac.uk/cosmic), among which, the most frequent (R678Q) was shown to enhance HER2 activity (43). It is tempting to speculate that mutations lying in this sequence would result in HER2 activation through interference with ERM binding. Taken together, these results demonstrate that ERM proteins are key allosteric regulators of HER2 activation, most likely by exerting a constraint on the juxtamembrane region of HER2 that hampers stabilisation in activated dimers. This mechanism prevents spontaneous HER2 activation in physiological condition suggesting that ERM binding precludes HER2-dependent oncogenic transformation, whereas loss of Moesin expression concomitant to HER2 overexpression in HER2+ breast cancers further contributes to the oncogenic activation of HER2.

Interestingly, we found a highly significant inverse correlation between Moesin expression and HER2 status in both cell lines and primary tumors of different cancers, including breast, cervix, stomach, bladder, pancreas, colon, ovary and kidney, indicating a potent role of Moesin in the development of HER2-positive cancers. As no mutation in Moesin gene can account for its downregulation in HER2positive cancers, this observation strongly suggests that epigenetic mechanisms, which perturbations represent hallmarks of human cancer cells, may directly or indirectly promote silencing of Moesin expression in HER2-positive cancer cells. Among epigenetic deregulation described in human cancers are changes in patterns of DNA methylation (44), the transcription of long non-coding RNAs, or microRNA (miRNA) that regulate gene expression by repressing translation and/or by promoting degradation of their target mRNA (45). Future studies will aim at characterizing the mechanisms by which Moesin expression is repressed in HER2-positive cancer cells.

The restriction of HER2 activation through ERM binding prompted us to translate our findings into pharmacological intervention on HER2+ breast tumours. The design of a high throughput screening 
method based on this interaction to find Moesin-mimicking compounds allowed us to identify Zuclopenthixol, a neuroleptic belonging to the group of thioxanthene commonly used to treat acute episodes of mental disorders by blockade of dopamine receptors. As expected, this compound could bind the juxtamembrane region of HER2 and block HER2 activation. As a consequence, it specifically reduces HER2-dependent proliferation and HER2-positive breast tumour progression in vivo. Due to this original mode of action, Zuclopenthixol is active on several intrinsically altered forms of HER2 such as the p95 truncated form of HER2 expressed in up to $30 \%$ of patients that confers resistance to antibodies-based therapies, or oncogenic forms of HER2 with activating mutations in the kinase domain (V777L and V842I) conferring resistance to tyrosine kinase inhibitors. Finally, this compound, able to rapidly penetrate the brain, shows significant activity on the progression of aggressive HER2+ breast cancer-derived brain tumours. Interestingly, the closely related dopamine antagonist Flupenthixol had similar effects, while others such as Chlorprothixene did not interact with the juxtamembrane region of HER2, demonstrating a mechanism independent of their neuroleptic activity. These effects were observed at well tolerated doses of Zuclopenthixol hydrochloride (4 to $10 \mathrm{mg} / \mathrm{kg}$ in mice), higher than recommended for chronic administration in humans (150-300 mg every 2 weeks). However, concentration may be further increased by steps of 50-100 mg once or twice weekly in patients requiring higher doses, or shorter intervals between doses. In addition, the time course of the antipsychotic action revealed a progressively enhanced response to antipsychotic drugs that follows an exponential curve with repeated drug administration (46). Given these advantages over existing therapies, Zuclopenthixol or Flupenthixol, would therefore provide a valuable treatment of HER2+ breast cancers and of a wide range of other HER2 overexpressing tumours, to efficiently combat primary tumours and derived-brain metastases in combination with the current HER2-targeted drugs.

Taken together, through the discovery of an unexpected allosteric regulation of HER2 activation by the ERM proteins, we identified Zuclopenthixol as an ERM mimicking compound. Our work reveals a new approach to target oncogenic forms of HER2. It provides the proof of concept that Zuclopenthixol possesses efficient anti-tumour activity in HER2+ primary cancers and brain metastases and could be used in addition to HER2 targeted therapies to prevent and treat HER2+ cancers. 


\section{ACKNOWLEDGEMENTS}

We thank B.B. Weksler, M. Rousset, M. Arpin, J. Delon, J. Baselga, M. Sliwkowski, H. Enslen and E Charafe-Jauffret for kindly providing reagents. We thank Remy Castellano and Yves Collette of the TrGET platform (Institut Paoli Calmettes) and Oncodesign for the animal experiments. We thank the Biophysical and Structural Chemistry platform of the Institut Européen de Chimie et Biologie (Pessac/Bordeaux, France) and the platforms of the Institut Cochin (Imag'IC, Cybio, HistIM) for their expert technical assistance. This work was supported by the Centre National de la Recherche Scientifique, the Institut National de la Santé et de la Recherche Médicale, the Université de Paris, the Association pour la Recherche contre le Cancer (SFI20101201780 and PJA 20131200363), The Fondation de France (00016391), the GEFLUC Association (GEFLUC2016) and the Société de Transfert de Technologies (SATT) Ile de France Innov (ANR-10-SATT-05-01). C Faure was supported by Fondation de France and SATT. R Djerbi-Bouillié was supported by a doctoral fellowship from the Ministère de la Recherche and by ARC. A Domingot was supported by a doctoral fellowship from the Ligue Contre le Cancer and ARC. S Bernard was supported by a doctoral fellowship from the Université Paris Diderot and ARC. 


\section{REFERENCES}

1. Du Z, Lovly CM. Mechanisms of receptor tyrosine kinase activation in cancer. Mol Cancer 2018;17:58

2. Montor WR, Salas A, Melo FHM. Receptor tyrosine kinases and downstream pathways as druggable targets for cancer treatment: the current arsenal of inhibitors. Mol Cancer 2018;17:55

3. Kreutzfeldt J, Rozeboom B, Dey N, De P. The trastuzumab era: current and upcoming targeted HER2+ breast cancer therapies. American journal of cancer research 2020;10:1045-67

4. Saez R, Molina MA, Ramsey EE, Rojo F, Keenan EJ, Albanell J, et al. p95HER-2 predicts worse outcome in patients with HER-2-positive breast cancer. Clin Cancer Res 2006;12:424-31

5. Chumsri S, Sperinde J, Liu H, Gligorov J, Spano JP, Antoine M, et al. High p95HER2/HER2 Ratio Associated With Poor Outcome in Trastuzumab-Treated HER2-Positive Metastatic Breast Cancer NCCTG N0337 and NCCTG 98-32-52 (Alliance). Clin Cancer Res 2018;24:3053-8

6. Connell CM, Doherty GJ. Activating HER2 mutations as emerging targets in multiple solid cancers. ESMO open 2017;2:e000279

7. Kodack DP, Askoxylakis V, Ferraro GB, Fukumura D, Jain RK. Emerging strategies for treating brain metastases from breast cancer. Cancer cell 2015;27:163-75

8. Ullrich A, Schlessinger J. Signal transduction by receptors with tyrosine kinase activity. Cell 1990;61:203-12

9. Olayioye MA, Neve RM, Lane HA, Hynes NE. The ErbB signaling network: receptor heterodimerization in development and cancer. Embo J 2000;19:3159-67.

10. Garrett TP, McKern NM, Lou M, Elleman TC, Adams TE, Lovrecz GO, et al. The crystal structure of a truncated ErbB2 ectodomain reveals an active conformation, poised to interact with other ErbB receptors. Mol Cell 2003;11:495-505

11. Di Fiore PP, Pierce JH, Kraus MH, Segatto O, King CR, Aaronson SA. erbB-2 is a potent oncogene when overexpressed in NIH/3T3 cells. Science 1987;237:178-82

12. Graus-Porta D, Beerli RR, Daly JM, Hynes NE. ErbB-2, the preferred heterodimerization partner of all ErbB receptors, is a mediator of lateral signaling. Embo J 1997;16:1647-55.

13. She QB, Chandarlapaty S, Ye Q, Lobo J, Haskell KM, Leander KR, et al. Breast tumor cells with PI3K mutation or HER2 amplification are selectively addicted to Akt signaling. PLoS One 2008;3:e3065

14. Junttila TT, Akita RW, Parsons K, Fields C, Lewis Phillips GD, Friedman LS, et al. Ligandindependent HER2/HER3/PI3K complex is disrupted by trastuzumab and is effectively inhibited by the PI3K inhibitor GDC-0941. Cancer cell 2009;15:429-40

15. Hughes SC, Fehon RG. Understanding ERM proteins--the awesome power of genetics finally brought to bear. Curr Opin Cell Biol 2007;19:51-6

16. Bretscher A, Edwards K, Fehon RG. ERM proteins and merlin: integrators at the cell cortex. Nat Rev Mol Cell Biol 2002;3:586-99

17. Yonemura S, Hirao M, Doi Y, Takahashi N, Kondo T, Tsukita S. Ezrin/radixin/moesin (ERM) proteins bind to a positively charged amino acid cluster in the juxta-membrane cytoplasmic domain of CD44, CD43, and ICAM-2. J Cell Biol 1998;140:885-95

18. Hamada K, Shimizu T, Yonemura S, Tsukita S, Hakoshima T. Structural basis of adhesionmolecule recognition by ERM proteins revealed by the crystal structure of the radixin-ICAM-2 complex. Embo J 2003;22:502-14

19. Mori T, Kitano K, Terawaki S, Maesaki R, Fukami Y, Hakoshima T. Structural basis for CD44 recognition by ERM proteins. The Journal of biological chemistry 2008;283:29602-12

20. Schweitzer KM, Vicart P, Delouis C, Paulin D, Drager AM, Langenhuijsen MM, et al. Characterization of a newly established human bone marrow endothelial cell line: distinct 
adhesive properties for hematopoietic progenitors compared with human umbilical vein endothelial cells. Lab Invest 1997;76:25-36

21. Chantret I, Rodolosse A, Barbat A, Dussaulx E, Brot-Laroche E, Zweibaum A, et al. Differential expression of sucrase-isomaltase in clones isolated from early and late passages of the cell line Caco-2: evidence for glucose-dependent negative regulation. J Cell Sci 1994;107 ( Pt 1):213-25

22. Gautreau A, Louvard D, Arpin M. Morphogenic effects of ezrin require a phosphorylationinduced transition from oligomers to monomers at the plasma membrane. J Cell Biol 2000;150:193-203

23. Lambotin M, Hoffmann I, Laran-Chich MP, Nassif X, Couraud PO, Bourdoulous S. Invasion of endothelial cells by Neisseria meningitidis requires cortactin recruitment by a phosphoinositide-3-kinase/Rac1 signalling pathway triggered by the lipo-oligosaccharide. J Cell Sci 2005;118:3805-16

24. Borg JP, Marchetto S, Le Bivic A, Ollendorff V, Jaulin-Bastard F, Saito $H$, et al. ERBIN: a basolateral PDZ protein that interacts with the mammalian ERBB2/HER2 receptor. Nat Cell Biol 2000;2:407-14

25. Shelly M, Mosesson Y, Citri A, Lavi S, Zwang Y, Melamed-Book N, et al. Polar expression of ErbB-2/HER2 in epithelia. Bimodal regulation by Lin-7. Developmental cell 2003;5:475-86

26. Dillon C, Creer A, Kerr K, Kumin A, Dickson C. Basolateral targeting of ERBB2 is dependent on a novel bipartite juxtamembrane sorting signal but independent of the $C$-terminal ERBIN-binding domain. Mol Cell Biol 2002;22:6553-63

27. Fievet BT, Gautreau A, Roy C, Del Maestro L, Mangeat P, Louvard D, et al. Phosphoinositide binding and phosphorylation act sequentially in the activation mechanism of ezrin. J Cell Biol 2004;164:653-9

28. Rothe R, Liguori L, Villegas-Mendez A, Marques B, Grunwald D, Drouet E, et al. Characterization of the cell-penetrating properties of the Epstein-Barr virus ZEBRA trans-activator. The Journal of biological chemistry 2010;285:20224-33

29. Liu X, Abdelrahim M, Abudayyeh A, Lei P, Safe S. The nonsteroidal anti-inflammatory drug tolfenamic acid inhibits BT474 and SKBR3 breast cancer cell and tumor growth by repressing erbB2 expression. Molecular cancer therapeutics 2009;8:1207-17

30. Zavodovskaya M, Campbell MJ, Maddux BA, Shiry L, Allan G, Hodges L, et al. Nordihydroguaiaretic acid (NDGA), an inhibitor of the HER2 and IGF-1 receptor tyrosine kinases, blocks the growth of HER2-overexpressing human breast cancer cells. Journal of cellular biochemistry 2008;103:624-35

31. Schwartz MA. Integrins, oncogenes, and anchorage independence. J Cell Biol 1997;139:575-8

32. Witzel I, Oliveira-Ferrer L, Pantel K, Muller V, Wikman H. Breast cancer brain metastases: biology and new clinical perspectives. Breast cancer research : BCR 2016;18:8

33. Tanaka Y, Hirata M, Shinonome S, Torii M, Nezasa KI, Tanaka H. Distribution analysis of epertinib in brain metastasis of HER2-positive breast cancer by imaging mass spectrometry and prospect for antitumor activity. Scientific reports 2018;8:343

34. McClatchey Al, Fehon RG. Merlin and the ERM proteins--regulators of receptor distribution and signaling at the cell cortex. Trends Cell Biol 2009;19:198-206

35. Weinman EJ, Hall RA, Friedman PA, Liu-Chen LY, Shenolikar S. The association of NHERF adaptor proteins with g protein-coupled receptors and receptor tyrosine kinases. Annu Rev Physiol 2006;68:491-505

36. Jeong J, VanHouten JN, Dann P, Kim W, Sullivan C, Yu H, et al. PMCA2 regulates HER2 protein kinase localization and signaling and promotes HER2-mediated breast cancer. Proceedings of the National Academy of Sciences of the United States of America 2016;113:E282-90

37. Jeong J, Choi J, Kim W, Dann P, Takyar F, Gefter JV, et al. Inhibition of ezrin causes PKCalphamediated internalization of erbb2/HER2 tyrosine kinase in breast cancer cells. The Journal of biological chemistry 2019;294:887-901 
38. Jeong J, VanHouten JN, Kim W, Dann P, Sullivan C, Choi J, et al. The scaffolding protein NHERF1 regulates the stability and activity of the tyrosine kinase HER2. The Journal of biological chemistry 2017;292:6555-68

39. Zhang X, Gureasko J, Shen K, Cole PA, Kuriyan J. An allosteric mechanism for activation of the kinase domain of epidermal growth factor receptor. Cell 2006;125:1137-49

40. Jura N, Endres NF, Engel K, Deindl S, Das R, Lamers MH, et al. Mechanism for activation of the EGF receptor catalytic domain by the juxtamembrane segment. Cell 2009;137:1293-307

41. Red Brewer M, Choi SH, Alvarado D, Moravcevic K, Pozzi A, Lemmon MA, et al. The juxtamembrane region of the EGF receptor functions as an activation domain. Mol Cell 2009;34:641-51

42. Nagy P, Claus J, Jovin TM, Arndt-Jovin DJ. Distribution of resting and ligand-bound ErbB1 and ErbB2 receptor tyrosine kinases in living cells using number and brightness analysis. Proceedings of the National Academy of Sciences of the United States of America 2010;107:16524-9

43. Pahuja KB, Nguyen TT, Jaiswal BS, Prabhash K, Thaker TM, Senger K, et al. Actionable Activating Oncogenic ERBB2/HER2 Transmembrane and Juxtamembrane Domain Mutations. Cancer cell 2018;34:792-806 e5

44. You JS, Jones PA. Cancer genetics and epigenetics: two sides of the same coin? Cancer cell 2012;22:9-20

45. He L, Hannon GJ. MicroRNAs: small RNAs with a big role in gene regulation. Nat Rev Genet 2004;5:522-31

46. Li M. Antipsychotic-induced sensitization and tolerance: Behavioral characteristics, developmental impacts, and neurobiological mechanisms. Journal of psychopharmacology 2016;30:749-70 
Table 1: Compounds showing inhibition of HER2/FERME interaction in AlphaScreen ${ }^{\circledR}$.

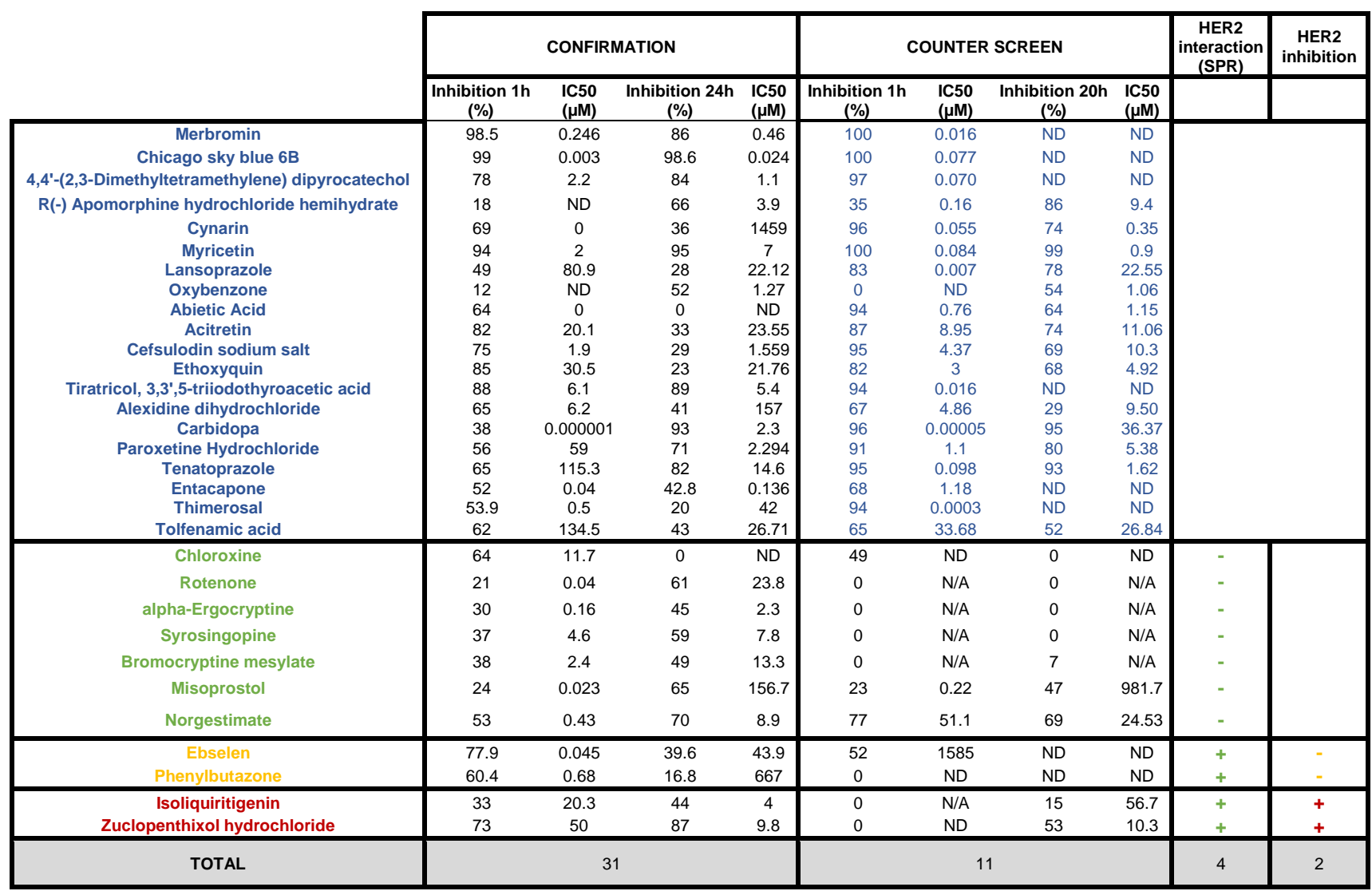

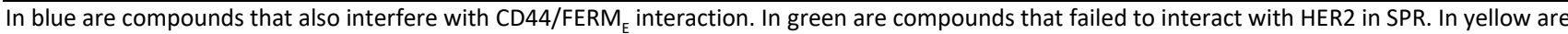
compounds that did not significantly inhibited HER2 activation. In red are compounds that selectively interfered with HER2/ FERM $\mathrm{E}_{\mathrm{E}}$ interaction, interacted with HER2 in SPR, and inhibited HER2 activation in HER2-positive breast cancer cells. ND, non determined. 


\section{FIGURE LEGENDS}

Figure 1: HER2 interaction with the ERM proteins Ezrin and Moesin addresses HER2 in ERM-enriched regions and control its ligand-independent activation

(A) Top, consensus sequence of the ERM binding motif and sequence alignment of the cytoplasmic juxtamembrane regions of adhesion molecules binding ERM proteins and of the HER family members. Basic and acidic residues are shown in blue and red, respectively. The basic clusters located in the ERM binding motif are underlined. Glutamines are shown in green. Key residues of the ICAM-2 binding motif to Radixin FERM domain are boxed in brown. Bottom, schematic depiction of gD-tagged HER2 vectors showing the transmembrane domain (TM) and the juxtamembrane region (JM) of gD-tagged wild type HER2 (gD-HER2-WT), and the mutant generated with alanine and glycine substitutions within the ERM binding motif (gD-HER2-EBM*).

(B) Human endothelial cells (HBMECs) were transfected with vectors encoding gD-HER2-WT or gDHER2-EBM*. Lysates were immunoprecipitated with an anti-gD antibody and analysed by western blot using anti-Moesin, anti-Ezrin and anti-gD antibodies.

(C) HBMECs were transfected with gD-HER2-WT or gD-HER2-EBM* constructs and cell lysates were analysed by western blot with anti-gD antibody (left panel). Decreasing concentrations of respective cell lysates were pulled down with GST-FERME and analysed by western blot with anti-gD and anti-GST antibodies (right panels).

(D) On the left, AlphaScreen ${ }^{\circledR}$ assay with 125 nM GST-FERME (red), or GST (blue) incubated with 10 nM biotinylated JM HER2 peptide (biot-JM HER2). A representative experiment is shown $(n=3)$ where data are mean $\pm \operatorname{sem}(n=2)$. On the right, AlphaScreen ${ }^{\circledR}$ assay with $83 \mathrm{nM}$ GST-FERME incubated in the presence (red) or in the absence (blue) of $9.25 \mathrm{nM}$ biot-JM HER2 peptide. Data are mean $\pm \operatorname{sem}(n=2)$.

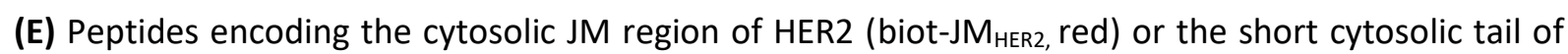
CD147 (cyto ${ }_{\mathrm{CD} 147}$, blue) were captured on a streptavidin-coated sensor chip (surface immobilization level of 60 and 115 resonance units (RU), depending on their respective molecular weight). GST (dotted lines) or GST-FERM (solid lines) were used as analytes and injected sequentially at $40 \mathrm{nM}, 200 \mathrm{nM}$ and $1000 \mathrm{nM}$ to perform single-cycle kinetics.

(F) Top panels, confluent monolayers of Caco-2/TC7 enterocytes were labelled with anti-Z01 (red) and anti-HER2 (green) antibodies (top panels) or with anti-Ezrin (red) and anti-HER2 (green) antibodies (lower panels). Images of $x y / x z$ plans and xz sections (z-cut) are shown. Merged images of the same fields are presented in the right panel (overlay) and higher magnifications of the inset are presented in the bottom panels. Horizontal black arrows indicate the lines on the xy plan where the z-cut presented underneath were selected. Bars represent $10 \mu \mathrm{m}$. Bottom panels, LLC-PK1 epithelial cells transfected with vectors encoding gD-HER2-WT or gD-HER2-EBM* were labelled with anti-Ezrin (red) 
and anti-HER2 (green) antibodies. Images of $x y / x z$ plans and $x z$ sections (z-cut) are shown. Merged images of the same fields are presented in the last panel (overlay) and higher magnifications of the inset are presented in the bottom panels. Horizontal black arrows indicate the lines on the xy plan where the z-cut presented underneath were selected, and the vertical blue arrows on the $z$ cuts indicate cell junctions. Bars represent $10 \mu \mathrm{m}$.

(G) Lysates from HBMECs non-transfected (NT) or co-transfected with gD-HER2 together with GFP, Ezrin-GFP or Moesin-GFP were analysed by western blots using antibodies against pY1248-HER2, HER2, GFP, p-ERK and Clathrin.

(H) Lysates from HBMECs transfected with empty vector (Mock), gD-HER2-WT or gD-HER2-EBM* together with GFP or FERME-VSV-g were analysed by western blots using anti-phosphotyrosine (PY), anti-HER2 and anti-VSV-g antibodies.

(I) HBMECs were co-transfected with empty vector (Mock), gD-HER2-WT or EBM* alone (solid lines) or together with FERM $\mathrm{E}^{-V S V-g}$ (dotted lines) and their proliferation was analysed $24 \mathrm{~h}, 48 \mathrm{~h}$, and $72 \mathrm{~h}$ after transfection. Results are means \pm sem $(n=4)$ of a representative of three independent experiments.

(J) HBMECs were transfected with siRNA targeting Ezrin, Moesin or cortactin or untargeted siRNA sequence as a negative control, and analysed by western blot using antibodies against Ezrin, Moesin, cortactin, clathrin, phosphotyrosine (PY), HER2, p-Akt or Akt.

(B-J) Shown are representative images or western blots of 2-3 independent experiments.

Figure 2: The unbalance in ERM/HER2 expression contributes to HER2 activation in HER2+ breast cancers

(A) Color-coded representation of HER2, Moesin (MSN), Ezrin (EZR) or Radixin (RDX) mRNA expression levels in 70 commonly used breast cancer cell lines from E-MTAB-2706 study (https://www.ebi.ac.uk/arrayexpress/). Spearman correlation coefficients $r=-0.4771$ (MSN), r=0.3354 (EZR), $r=-0.2542$ (RDX); $P$ value (two-tailed), $P<0.0001$ (MSN), $P=0.0045$ (EZR), $P=0.337$ (RDX). Cell lines used in this study are highlighted in grey.

(B) Lysates from breast cancer cell lines (MDAMB-231, SKBR3, BT474 and HCC1954) were analysed by western blots using anti-phospho HER2 ( $\mathrm{pY}_{1248}$-HER2), anti-HER2, anti-Moesin or anti-Ezrin antibodies. (C) Color-coded representation of the mRNA expression level of HER2, Moesin (MSN), Ezrin (EZR) and Radixin (RDX) in samples from 526 patients with breast invasive carcinoma from TCGA cohort (http://www.cbioportal.org). Spearman correlation coefficients $r=-0.3010$ (MSN), $r=0.1538$ (Ezrin), $r=-$ 0.1783 (Radixin); $\mathrm{P}$ value (two-tailed) $\mathrm{P}<0.0001, \mathrm{P}=0.0004$ (Ezrin), $\mathrm{P}<0.0001$ (Radixin).

(D) Representative images of human breast carcinoma sections from each molecular subtype labelled by immunohistochemistry with anti-HER2 (brown) and anti-Moesin or anti-Ezrin (red) antibodies. TNBC $=$ triple negative breast cancers. Bars represent $50 \mu \mathrm{m}$. The immunohistochemistry analysis 
performed on 67 cases of breast ductal carcinoma with known molecular subtypes is shown in supplementary figure 4.

(E) Lysates of BT474 cells mock transfected, transfected with a vector encoding GFP, or with increasing concentrations of vectors encoding Ezrin-GFP or FERME-GFP, were analysed by western blots with antiphosphotyrosine (PY) and anti-HER2 antibodies. Shown is a representative blot of $n=3$ independent experiments.

(F) BT474 cells transfected with GFP, Ezrin-GFP, FERME-GFP or Moesin-GFP were monitored by timelapse imaging (Incucyte). Quantification of the number of GFP-positive cells over time was performed (data presented in supplementary figure $6 \mathrm{~B}-\mathrm{C}$ ). Shown is the quantification of GFP-positive cells ( $\mathrm{n}=12$ fields) $72 \mathrm{~h}$ post-transfection, where data are mean \pm sem $(n=2)$; one-way ANOVA followed by Bonferroni's multiple comparison test, ${ }^{* * * *} \mathrm{P}<0.0001$.

Figure 3: Zuclopenthixol is an ERM-mimicking compound conferring allosteric inhibition of HER2 (A) Screening strategy used to identify Moesin mimicking compounds. 1463 drugs from commercially available libraries were screened for their ability to compete with $H E R 2 / F E R M_{E}$ interaction leading to the identification of 77 first hits. After removal of the false positive, 31 remaining compounds were submitted to a counter-screen to select hits showing no competition with CD44/FERME interaction. 11 hits were characterized for their ability to bind peptide encoding the JM domain of HER2 in SPR, to selectively inhibit HER2 activation and HER2-dependent cell proliferation and for their absence of toxicity on cells that do not overexpress HER2. The best hit (Zuclopenthixol) was tested in vivo in an orthotopic xenograft model.

(B) Signal of HER2/FERM interaction $24 \mathrm{~h}$ after the addition of compounds (Zuclopenthixol is shown in red).

(C) Proliferation curve of HER2+ (SKBR3 or BT474) or HER2-negative (MDAMB-231) breast cancer cell lines treated with vehicle or with $3 \mu \mathrm{M}$ Zuclopenthixol assessed by real time impedance-based cell proliferation assay. Arrows indicate beginning of the treatment. A representative experiment is shown $(n=2-4)$ where data are mean $\pm S D(n=2-4)$; statistical analysis are paired t test, $* * * * P<0.0001$.

(D-F) Anchorage-independent growth of SKBR3 (D), BT474 (E) or MDAMB-231 (F) cells treated with vehicle or $5 \mu \mathrm{M}$ Zuclopenthixol. Representative images and quantification of the colony mean area are shown where data are mean $\pm \operatorname{sem}(n=2)$, unpaired t test, $* * P<0.01(n=5-25)(D) ;(n=1)$, unpaired $t$ test, * $P<0.05$ ( $n=2-5$ replicates) (F); $(n=1)$, unpaired t test, $n s \quad P=0.59 .8$ ( $n=2-4$ replicates) (F). 
Figure 4: Zuclopenthixol inhibits the HER2-dependent tumour growth in orthotopic xenograft model (A-J) $5.10^{6}$ BT474 cells were implanted orthotopically in the mammary fat pad of NOD.Cg-Prkdc scid/J mice, in the presence of estradiol supplement. After 19 days, mice were randomized in 3 groups ( $n=8$ or 9 mice/group) and administered intraperitoneally with Zuclopenthixol ( $4 \mathrm{mg} / \mathrm{kg}$ per day for 5 days a week $n=8$, or $5 \mathrm{mg} / \mathrm{kg}$ per day for 3 days a week; $n=9$ ) or vehicle ( $10 \%$ DMSO in PBS; $n=9$,) during 3 weeks.

(A) Scheme of the protocol used in vivo.

(B) Individual tumour volume of vehicle-treated or Zuclopenthixol-treated mice. Arrow indicate beginning of the treatment at Day 19.

(C) Mean tumour volume of vehicle-treated or Zuclopenthixol-treated mice, normalized to the tumour volume at Day 19. Data are mean \pm sem; two-way RM ANOVA followed by Bonferroni's multiple comparison test, $* * \mathrm{P}<0.01, * * * \mathrm{P}<0.001, * * * * \mathrm{P}<0.0001$.

(D) Tumours collected at the end of the experiment.

(E) Scatter plot showing tumour weight at the end of the experiment and mean \pm sem; one-way ANOVA followed by Bonferroni's multiple comparison test, * $\mathrm{P}<0.05$.

(F) Mean weight of mice monitored 3 times a week during the treatment.

(G) Western blot analysis of the tumour lysates from vehicle- or Zuclopenthixol-treated mice $(4 \mathrm{mg} / \mathrm{kg})$ using antibodies directed against activated HER2 (pY HER2), activated ERK (pERK), activated Akt (pAkt) or Clathrin as a loading control.

(H) Quantification of the results presented in $\mathrm{G}$ where data are mean $\pm \operatorname{sem}(n=4)$, multiple paired $t$ test $* \mathrm{P}<0.05$.

(I) Representative images of immunohistochemistry analysis of tumour sections from vehicle- or Zuclopenthixol-treated mice using antibodies directed against HER2 (brown) and Ki67 (red). Bars represent $100 \mu \mathrm{m}$.

(J) For quantitative analysis, sections were also labelled in immunofluorescence and the proliferation index (KI67/DAPI) is displayed on the right where data are mean \pm sem $(n=2-4)$; unpaired t test **** $\mathrm{P}$ $<0.0001$.

Figure 5: Zuclopenthixol reduces the growth of HER2-dependent brain tumours

(A-D) $1.10^{5}$ human breast cancer BT474 cells were intracranially implanted in immunodeficient BALB/C Nude mice supplemented in estradiol. 10 days after (D10), tumour volume was evaluated by MRI and mice were randomized to be treated with Zuclopenthixol (M, Tu: $7 \mathrm{mg} / \mathrm{kg} ; \mathrm{W}-\mathrm{F}: 10 \mathrm{mg} / \mathrm{kg} \mathrm{IP;}=10$ ) or with vehicle ( $20 \% \mathrm{w} / \mathrm{v}$ hydroxypropyl- $\beta$-cyclodextrin (HP- $\beta-C D)$ IP; $n=10) .10$ days after (D20) another MRI was performed to monitor brain tumour growth.

(A) Scheme of the protocol used in vivo. 
CAN-21-0162R Faure et al

(B) Representative images of the MRI analysis at D10 and D20.

(C) Individual tumour volume between D10 and D20.

(D) Mean tumour volume of vehicle-treated or Zuclopenthixol-treated mice, normalized to the tumour volume at $\mathrm{D} 10$. Data are mean \pm sem; unpaired t test, * $\mathrm{P}=0.0112$. 

$A_{\begin{array}{r}\text { binding } \\ \text { motif }\end{array}} \underset{\begin{array}{c}\text { bRM } \\ \text { basic region }\end{array}}{\begin{array}{c}\text { consensus } \\ \text { sequence }\end{array}} \begin{gathered}\mathrm{C} \text {-ter } \\ \text { basic region }\end{gathered}$

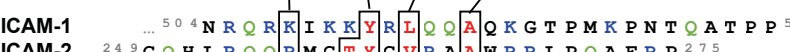

ICAM-2 249 G $Q$ HLR $Q Q$ R M G T Y G VRA A R R R L P $Q$ AFRP ${ }^{2}$

CAM-3 REH

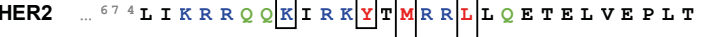

EGFR …64 LFMRRRHIVRKRTITRLLQEREIVEPIT 660

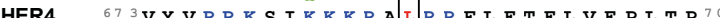

${ }_{\text {gD-HER2-WT }}^{{ }^{670}{ }_{\text {VFGILIKRRQQKIRKYTMRRILQETELVEPLTPSGAMPNQA }}{ }^{710}}$

$\begin{array}{ll}\text { gD-HER2-WT } & \text { VFGILIKRRQQKIRKYMRRILQETELVEPLTPSGAMPNQA } \\ \text { gD-HER2-EBM* } & \text { VFGILIGGGQOKIGKATMRRILOETELVEPLTPSGAMPNQA }\end{array}$

$\mathrm{F}$
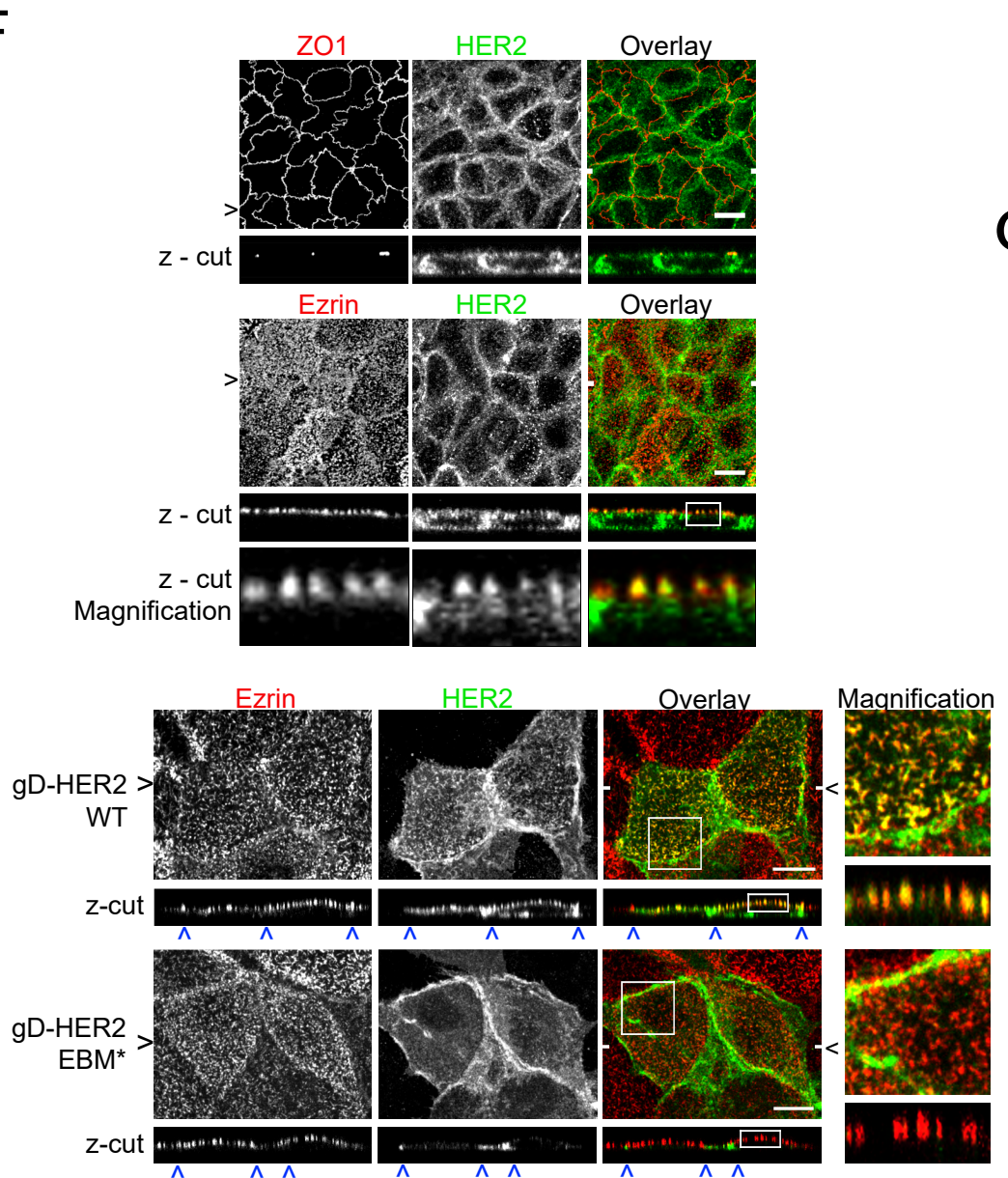

B

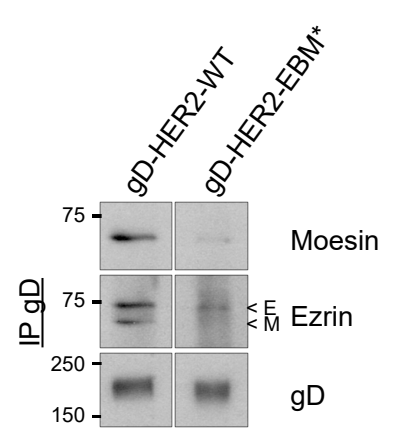

$\mathrm{D}$

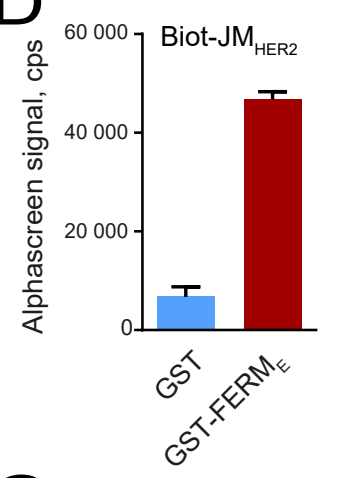

G
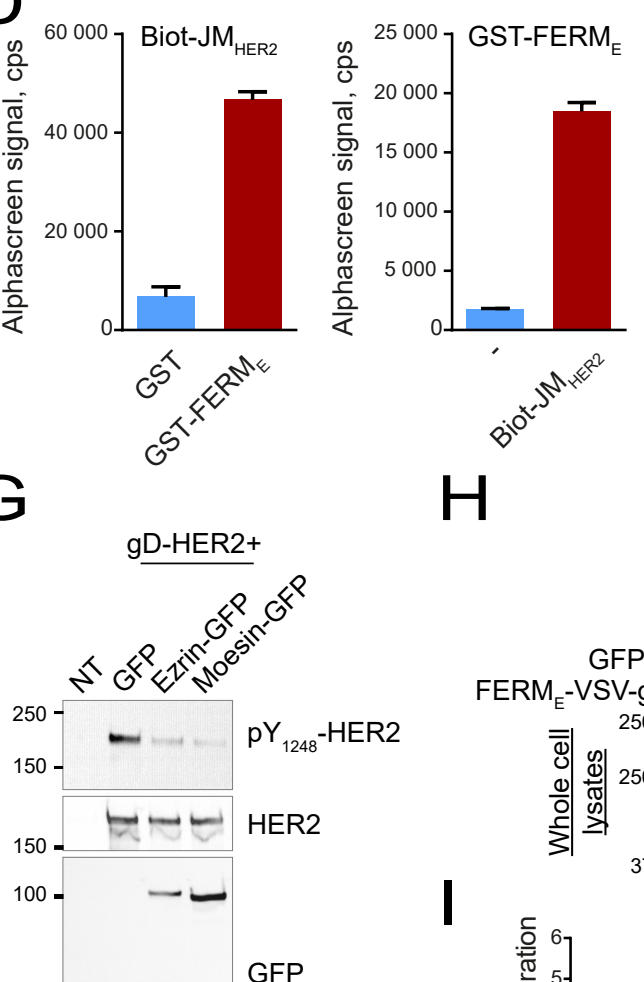

$\mathrm{H}$
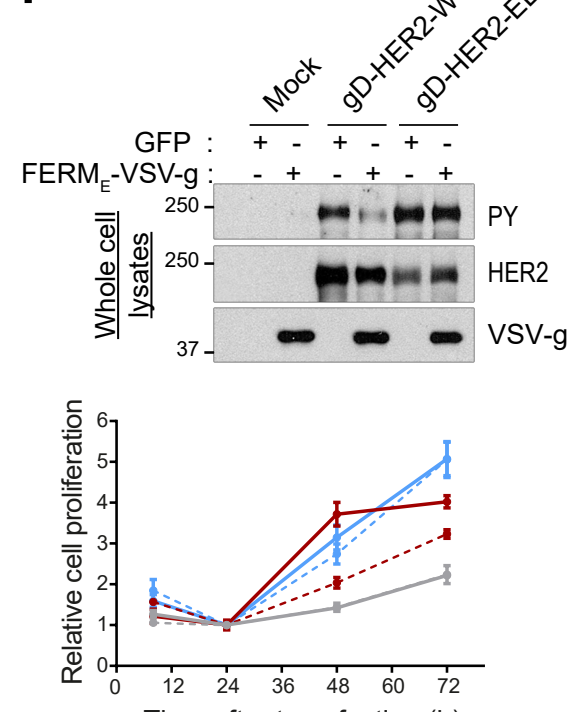

Time after transfection $(\mathrm{h})$

- Empty vector

-. Empty vector + FERM - -VSV-g

$\rightarrow$ gD-HER2-WT

- gD-HER2-WT + FERM - -VSV-g

$\rightarrow$ gD-HER2-EBM*

- gD-HER2-EBM* + FERM ${ }_{\mathrm{E}}-\mathrm{VSV}-\mathrm{g}$
- Biot--Cyto ${ }_{\text {CD147 }} \mid$ GST-FERM \begin{tabular}{l|l} 
- & Biot-JM \\
- Biot-Cyto & BD147 \\
Biot
\end{tabular}

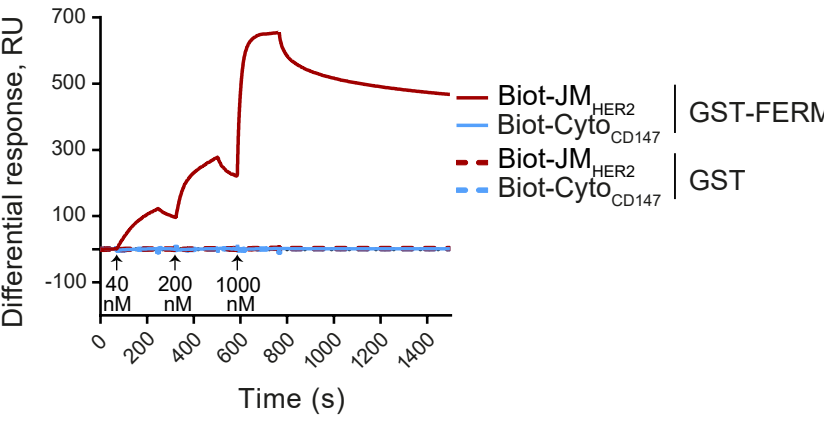


A

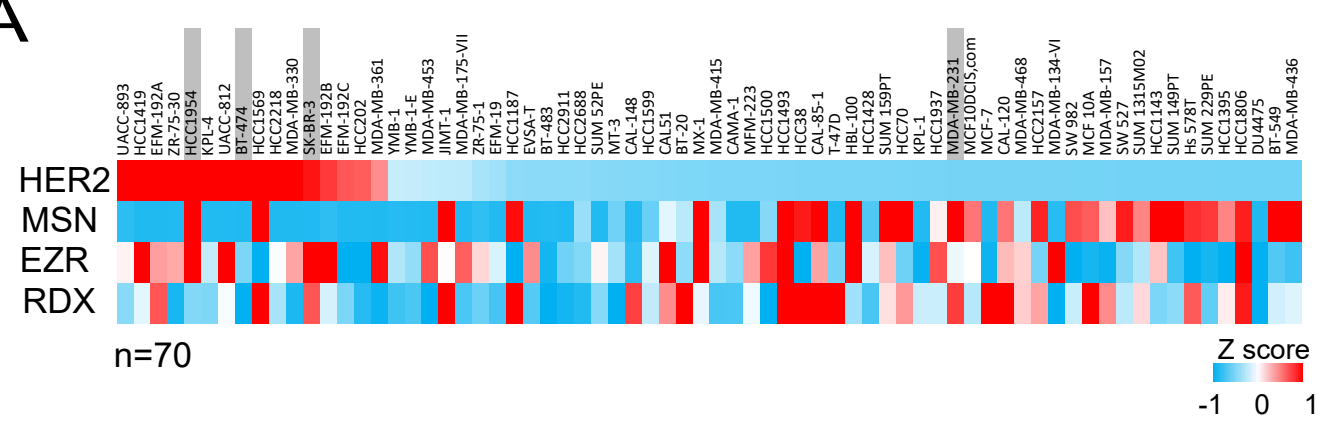

B

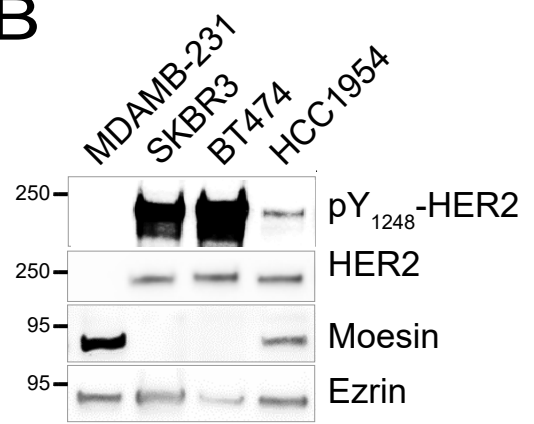

C

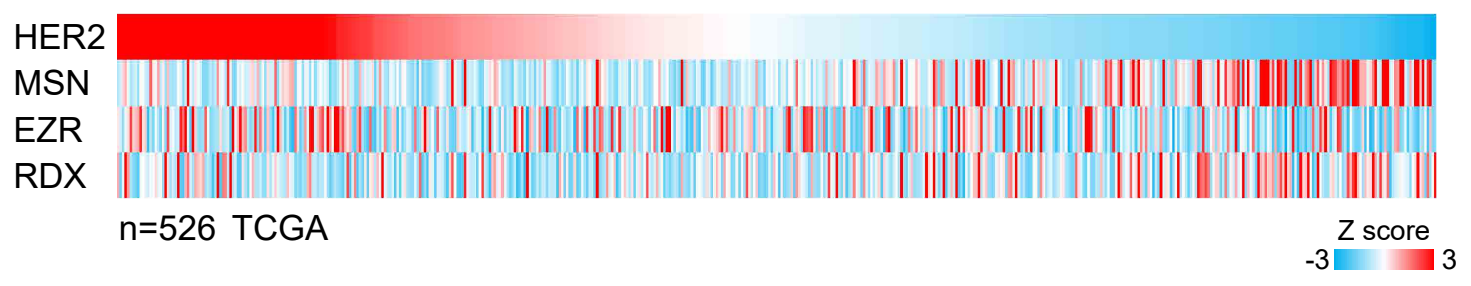

D

TNBC

Luminal A

Luminal $B$

HER2

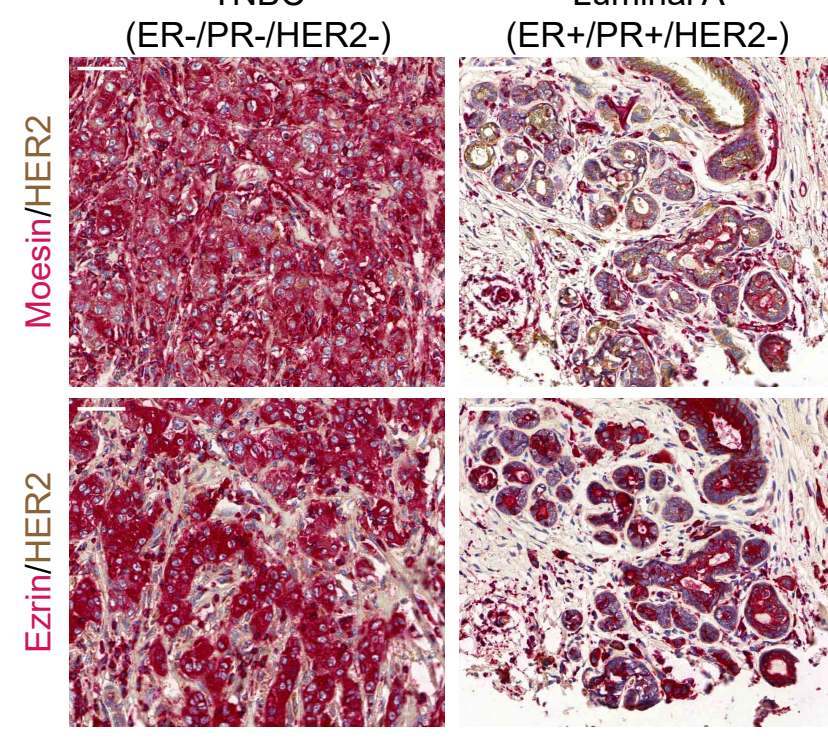

(ER+/PR+/HER2+)

(ER-/PR-/HER2+)
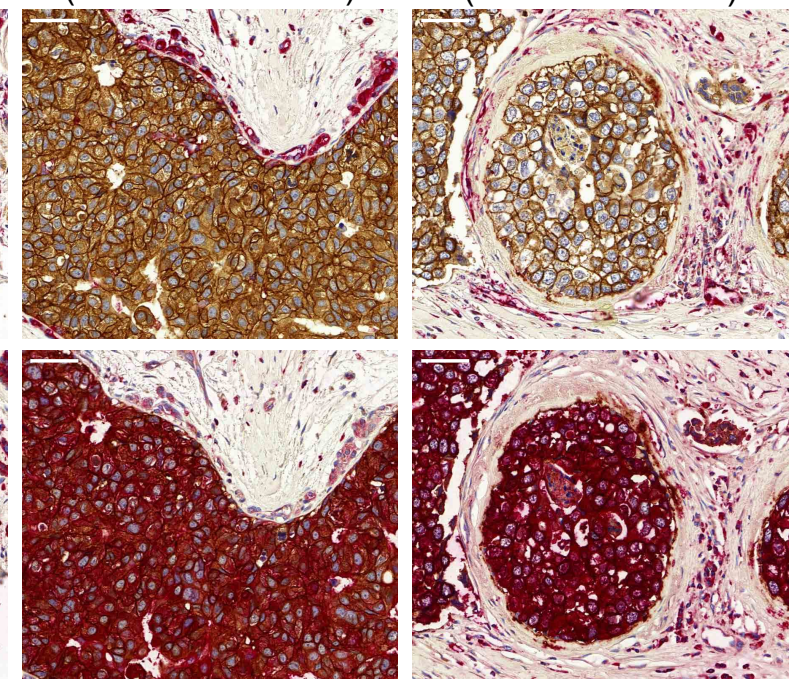

E

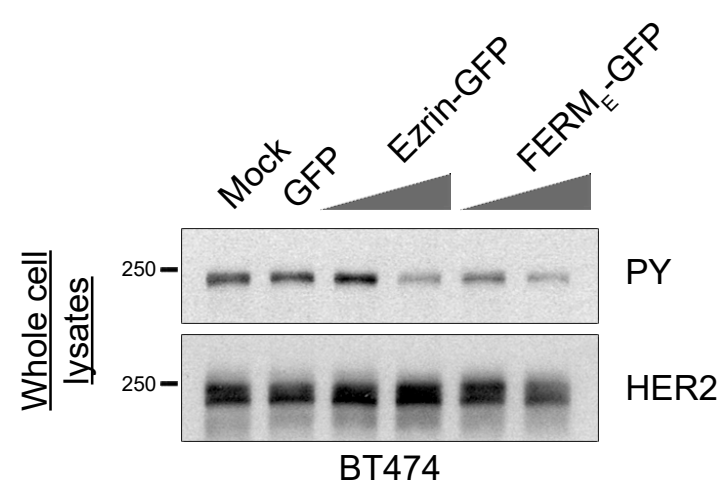

F

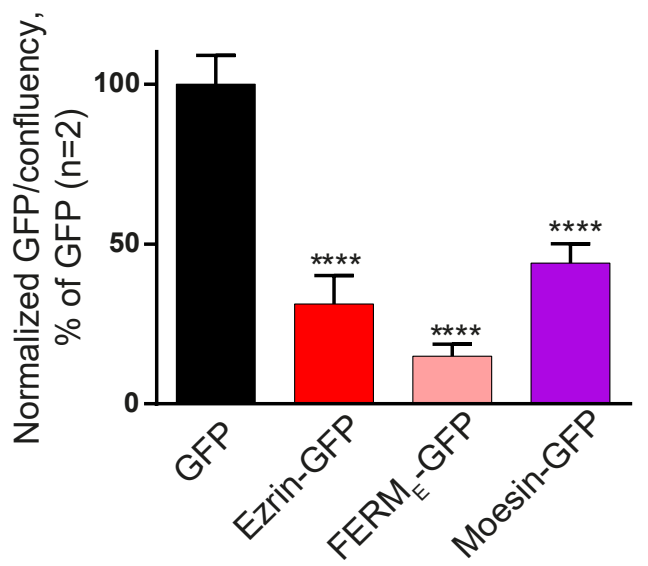

Figure 2 
A

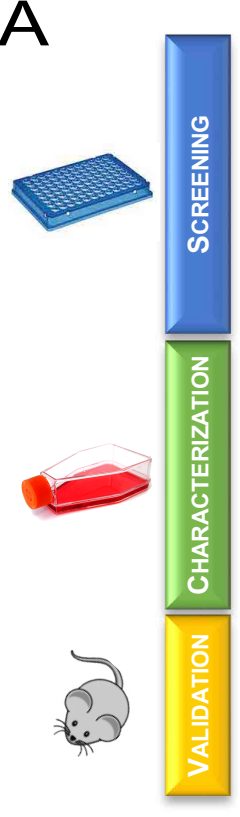

1463 compounds

B

77

55

Confirmation

31

Counter screen

No competition with CD44-FERM interaction

EBM binding (SPR)

HER2 inhibition

Specificity \& Toxicity

Inhibition of tumour growth in vivo

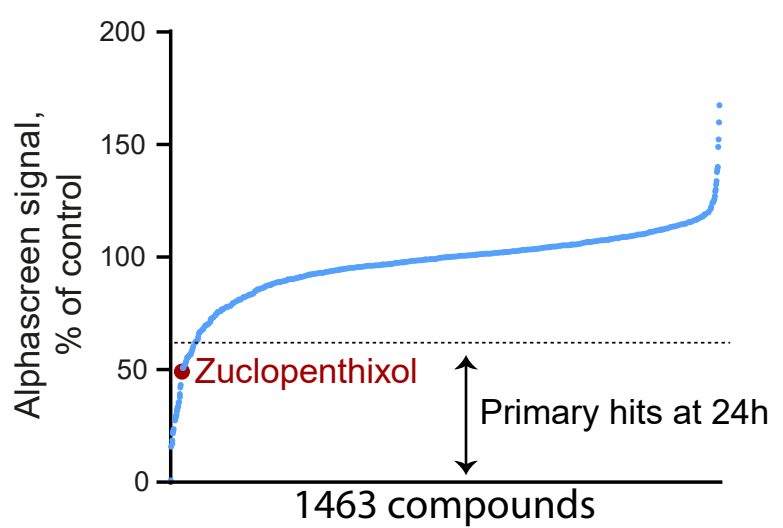

1 Zuclopenthixol

C

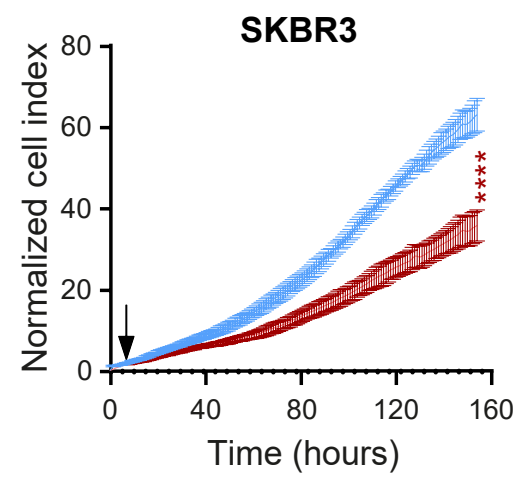

$\mathrm{D}$

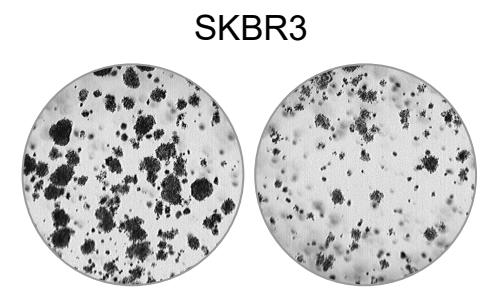

Vehicle Zuclopenthixol

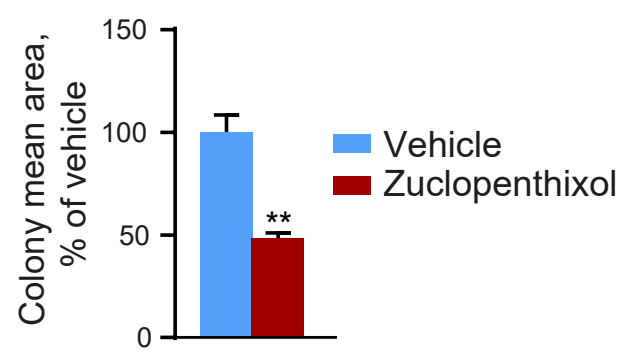

Vehicle Zuclopenthixol $3 \mu \mathrm{M}$

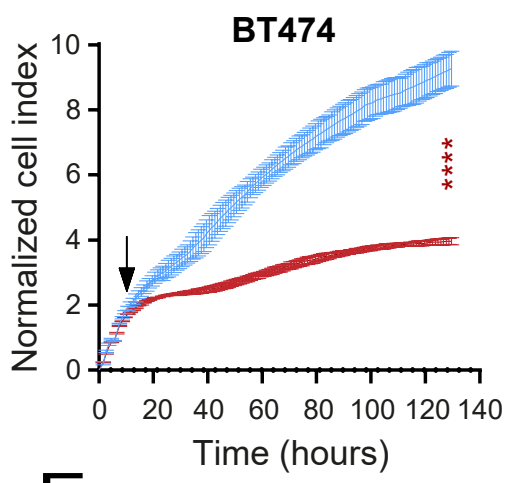

$E$

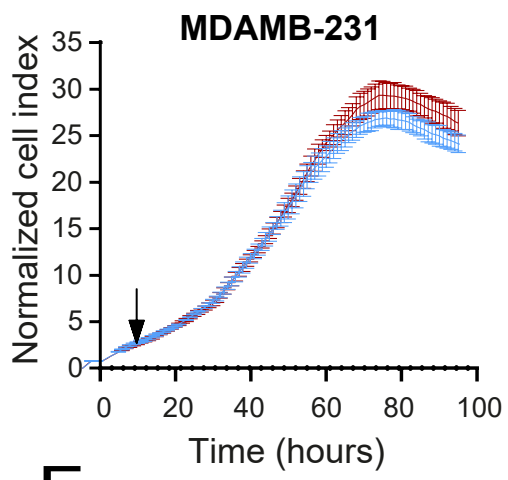

BT474

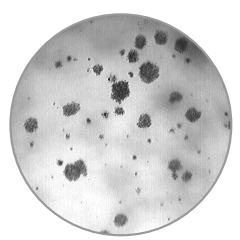

Vehicle

Zuclopenthixol

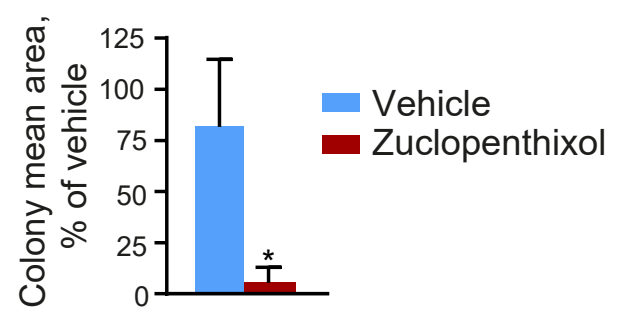

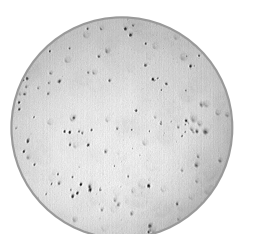

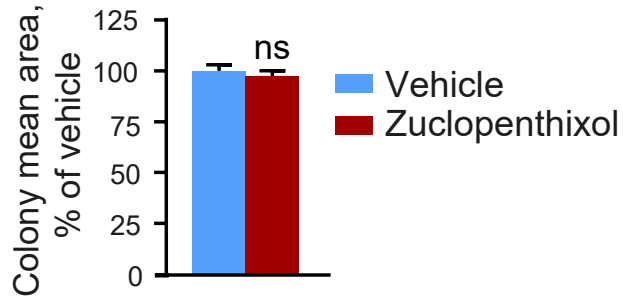


A

B
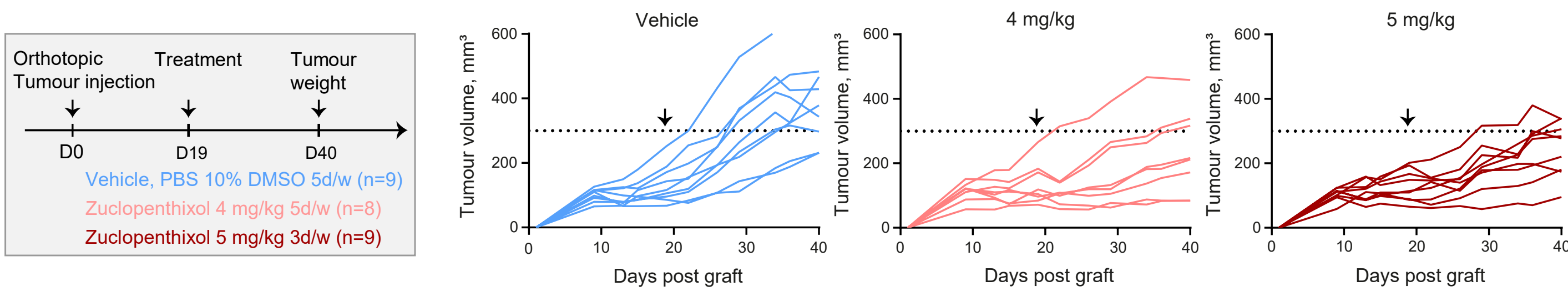

C

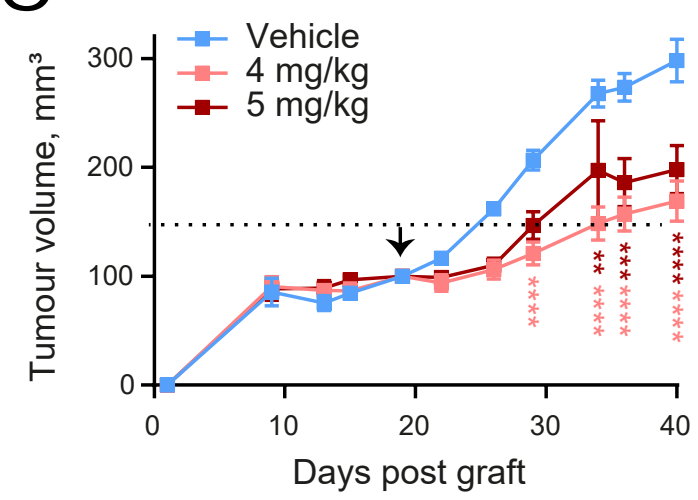

G
D

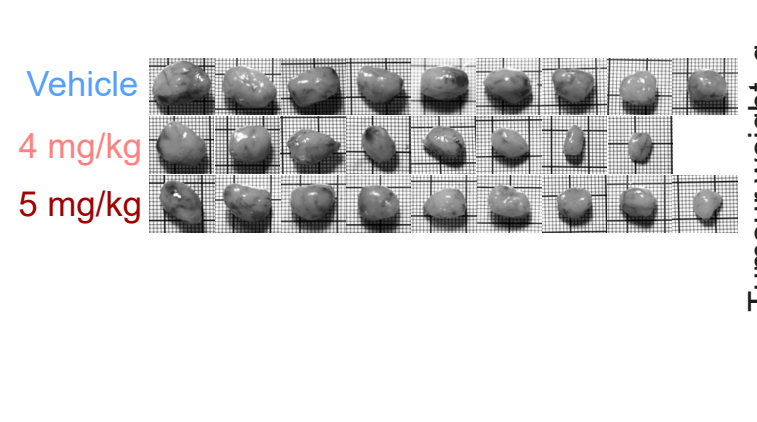

$\mathrm{H}$

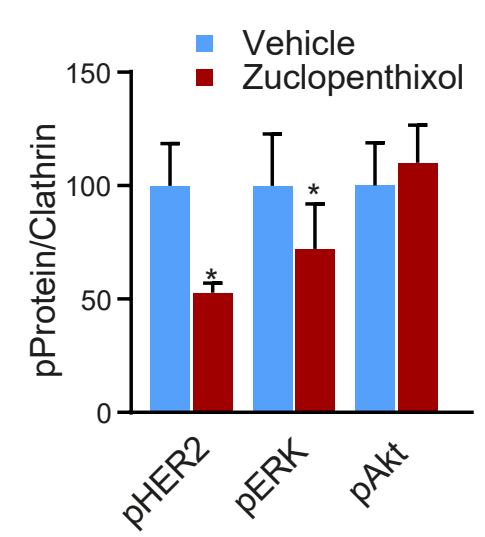

E

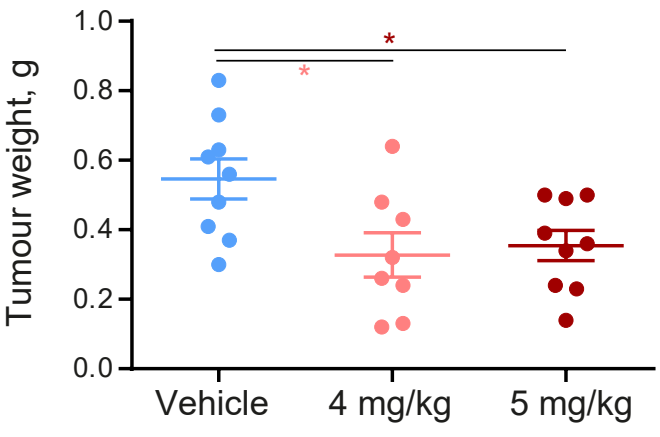

I

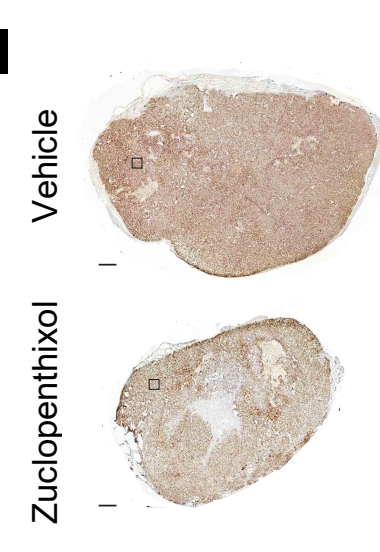

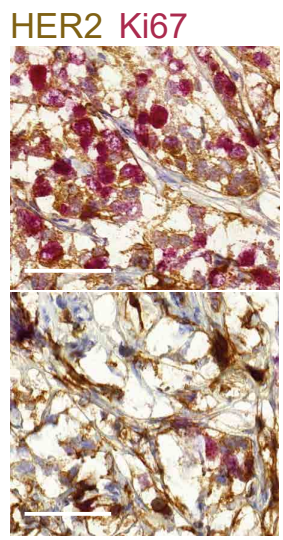

\section{F}

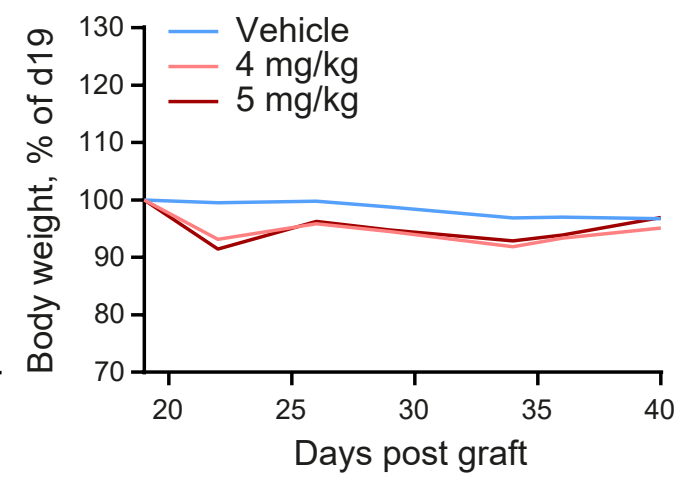

$J$

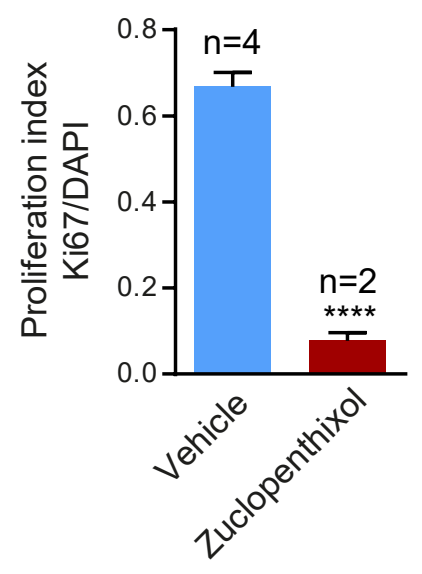

Figure 4 
A

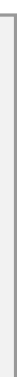

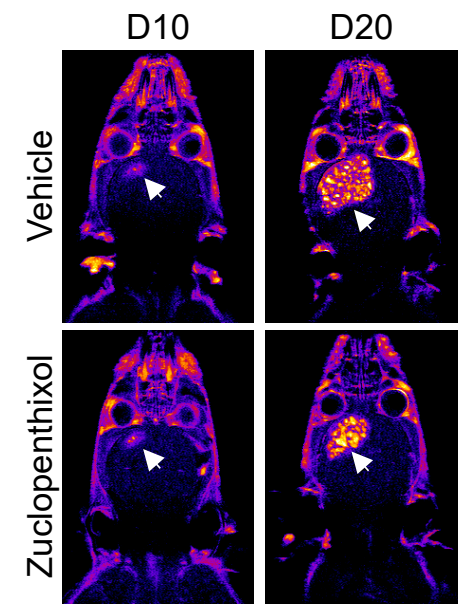

C
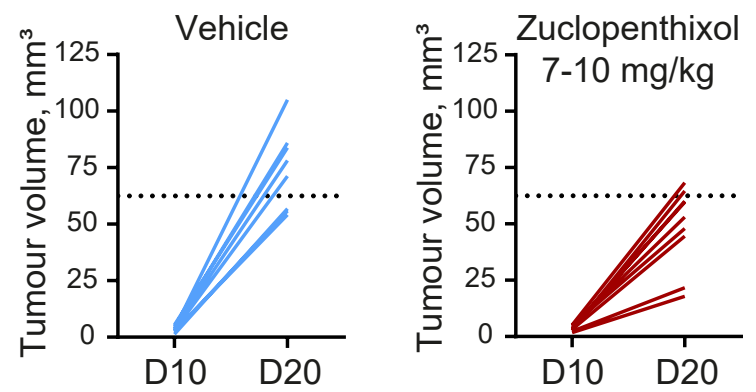

D

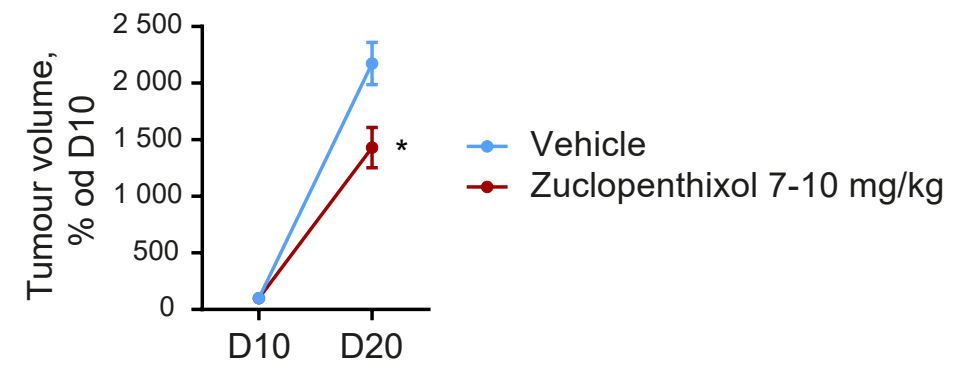

Figure 5 